\title{
METASTABILITY OF REVERSIBLE RANDOM WALKS IN POTENTIAL FIELDS
}

\author{
C. LANDIM, R. MISTURINI, K. TSUNODA
}

\begin{abstract}
Let $\Xi$ be an open and bounded subset of $\mathbb{R}^{d}$, and let $F: \Xi \rightarrow \mathbb{R}$ be a twice continuously differentiable function. Denote by $\Xi_{N}$ the discretization of $\Xi, \Xi_{N}=\Xi \cap\left(N^{-1} \mathbb{Z}^{d}\right)$, and denote by $X_{N}(t)$ the continuous-time, nearest-neighbor, random walk on $\Xi_{N}$ which jumps from $\boldsymbol{x}$ to $\boldsymbol{y}$ at rate $e^{-(1 / 2) N[F(\boldsymbol{y})-F(\boldsymbol{x})]}$. We examine in this article the metastable behavior of $X_{N}(t)$ among the wells of the potential $F$.
\end{abstract}

\section{INTRODUCTION}

We introduced recently in 2, 3] an approach to prove the metastable behavior of Markov chains which has been successfully applied in several different contexts. We refer to [4, 18, for a description of the method and for examples of Markov chains whose metastable behavior has been established with this approach.

We examine in this article the metastable behavior of reversible random walks in force fields. This is an old problem whose origin can be traced back at least to Kramers [17. It has been adressed by Freidlin and Wentsell [14 and by Galves, Olivieri and Vares [15] in the context of small random perturbations of dynamical systems, and, more recently, by Bovier, Eckhoff, Gayrard and Klein in a series of papers [7, 8, 9, 10, through the potential theoretic approach. This problem has raised interest and has found applications in many areas, as computer sciences [11] and chemical physics 20 .

The first main result of this article, Theorem 2.4 states that starting from a neighborhood of a local minimum of the force field, in an appropriate time-scale, the evolution of the random walk can be described by a reversible Markov chain in a finite graph, in which the vertices represent the wells of the force field and the edges the saddle points.

More precisely, denote by $X_{N}(t)$ a reversible random walk evolving in a discretization of a bounded domain $\Xi \subset \mathbb{R}^{d}$ according to a force field $F: \Xi \rightarrow \mathbb{R}$. A precise definition of the dynamics is given below in 2.1). Let $\boldsymbol{x}_{1}, \ldots, \boldsymbol{x}_{L}$ be the local minima of the field $F$, and let $Y_{N}(t)$ be the process which records the minima visited: $Y_{N}(t)$ is equal to $j$ if the chain $X_{N}(t)$ belongs to a neighborhood of $\boldsymbol{x}_{j}, 1 \leq j \leq L$, and 0 otherwise. Clearly, $Y_{N}(t)$ is not Markovian. Theorem 2.4 asserts that starting from a neighborhood of a local minimum $\boldsymbol{x}_{j}$, there exists a time scale $\beta_{N}$, which depends on $j$, in which $Y_{N}\left(t \beta_{N}\right)$ converges in some topology to a Markovian dynamics whose state space is a subset of $\{1, \ldots, L\}$. This asymptotic dynamics may have absorbing points, and its jump rates depend solely on the behavior of the potential in the neighborhoods of the local minima and in the neighborhoods of the saddle points. Theorem 2.4 is similar in spirit to the one of

Key words and phrases. Reversible random walks, Metastability, Exit points. 
Noé, Wu, Prinz and Plattner [20], who proved that projected metastable Markovian dynamics can be well approximated by hidden Markovian dynamics.

The second main result, Theorem 2.7, adresses the problem of the exit points from a domain. Consider a local minimum $\boldsymbol{x}_{j}$ of the force field and denote by $\left\{\boldsymbol{z}_{1}, \ldots, \boldsymbol{z}_{K}\right\}$ the lowest saddle points of $F$ which separate $\boldsymbol{x}_{j}$ from the other local minima. Theorem 2.7 provides the asymptotic probabilities that the chain $X_{N}(t)$ will traverse a mesoscopic neighborhood of a saddle point $\boldsymbol{z}_{i}$ before hitting another local minima of the force field.

We explained already in 4 the main differences between our approach and the potential theoretic one [7, 8, and between our approach and the pathwise one due to Cassandro, Galves, Olivieri and Vares [12. We will not repeat this exposition here. Our approach does not aim to characterize the typical paths in a transition between two metastable states, in contrast with the transition path theory [13. Nevertheless, in the case where the number of wells is small, as in the examples presented in [19], Theorems 2.4 and 2.7 describe the distribution of the transition paths, at least at the scale of the metastable sets, by indicating the sequence of metastable sets visited in a transition between two metastable sets.

In the case of complex networks, the Lennard--Jones clusters analyzed in 11 for instance, to give a rough view of the transition paths from two metastable states, we may proceed in two ways. One possibility is to reduce the number of nodes by considering the trace of the original chain on a subset of the state space (cf. [2, Section 6.1] for the definition of trace processes). Avena and Gaudillière [1 proposed a natural algorithm to reduce the number of vertices of a chain. The algorithm produces a subset $V$ with the property that the mean hitting time of $V$ does not depend on the starting point. In this sense the vertices of $V$ are "uniformly" distributed among the set of nodes. The algorithm can also be calibrated to provide a large or small set of nodes $V$.

Another possibility is to identify certain nodes, losing the Markov property, and to apply Theorem 2.4 below to approximate this new dynamics by a Markovian dynamics. To describe the transition paths at this level of accuracy, one can compute for these reduced dynamics the equilibrium potential between two metastable sets (the committor in the terminology of [11]), and the optimal flow for Thomson's principle (the probability current of reactive trajectories).

In both cases, the selection of the set of nodes or the selection of nodes to be merged have to be carried out judiciously, to reduce as much as possible the number of nodes without losing the essential features of the original chain. From a computational point of view, the jump rates of trace process are easily calculated, while the jump rates of projected processes are more difficult to derive. In the first case, it suffices to apply recursively the first displayed equation below the proof of Corollary 6.2 in [2, while in the second case, one has to calculate the capacities between the metastable sets.

\section{Notation And Results}

Let $\Xi$ be an open and bounded subset of $\mathbb{R}^{d}$, and denote by $\partial \Xi$ its boundary, which is assumed to be a smooth manifold. Fix a twice continuously differentiable function $F: \Xi \cup \partial \Xi \rightarrow \mathbb{R}$, with a finite number of critical points, satisfying the following assumptions: 
(H1) The second partial derivatives of $F$ are Lipschitz continuous. Denote by $C_{1}$ the Lipschitz constant;

(H2) All the eigenvalues of the Hessian of $F$ at the critical points which are local minima are strictly positive.

(H3) The Hessian of $F$ at the critical points which are not local minima or local maxima has one strictly negative eigenvalue, all the other ones being strictly positive. In dimension 1 this assumption requires the second derivative of $F$ at the local minima to be strictly negative.

(H4) For every $\boldsymbol{x} \in \partial \Xi,(\nabla F)(\boldsymbol{x}) \cdot \boldsymbol{n}(\boldsymbol{x})<0$, where $\boldsymbol{n}(\boldsymbol{x})$ represents the exterior normal to the boundary of $\Xi$, and $\boldsymbol{x} \cdot \boldsymbol{y}$ the scalar product of $\boldsymbol{x} \boldsymbol{y} \in \mathbb{R}^{d}$. This hypothesis guarantees that $F$ has no local minima at the boundary of $\Xi$.

Denote by $\Xi_{N}$ the discretization of $\Xi: \Xi_{N}=\Xi \cap\left(N^{-1} \mathbb{Z}^{d}\right), N \geq 1$, where $N^{-1} \mathbb{Z}^{d}=\left\{\boldsymbol{k} / N: \boldsymbol{k} \in \mathbb{Z}^{d}\right\}$. The elements of $\Xi_{N}$ are represented by the symbols $\boldsymbol{x}=\left(\boldsymbol{x}_{1}, \ldots, \boldsymbol{x}_{d}\right), \boldsymbol{y}$ and $\boldsymbol{z}$. Let $\mu_{N}$ be the probability measure on $\Xi_{N}$ defined by

$$
\mu_{N}(\boldsymbol{x})=\frac{1}{Z_{N}} e^{-N F(\boldsymbol{x})}, \quad \boldsymbol{x} \in \Xi_{N}
$$

where $Z_{N}$ is the partition function $Z_{N}=\sum_{\boldsymbol{x} \in \Xi_{N}} \exp \{-N F(\boldsymbol{x})\}$. Let $\left\{X_{N}(t): t \geq\right.$ $0\}$ be the continuous-time Markov chain on $\Xi_{N}$ whose generator $L_{N}$ is given by

$$
\left(L_{N} f\right)(\boldsymbol{x})=\sum_{\substack{\boldsymbol{y} \in \Xi_{N} \\\|\boldsymbol{y}-\boldsymbol{x}\|=1 / N}} e^{-(1 / 2) N[F(\boldsymbol{y})-F(\boldsymbol{x})]}[f(\boldsymbol{y})-f(\boldsymbol{x})],
$$

where $\|\cdot\|$ represents the Euclidean norm of $\mathbb{R}^{d}$. The rates were chosen for the measure $\mu_{N}$ to be reversible for the dynamics. Denote by $R_{N}(\boldsymbol{x}, \boldsymbol{y}), \lambda_{N}(\boldsymbol{x}), \boldsymbol{x}$, $\boldsymbol{y} \in \Xi_{N}$, the jump rates, holding rates of the chain $X_{N}(t)$, respectively:

$$
\begin{aligned}
R_{N}(\boldsymbol{x}, \boldsymbol{y}) & = \begin{cases}e^{-(1 / 2) N[F(\boldsymbol{y})-F(\boldsymbol{x})]} & \|\boldsymbol{y}-\boldsymbol{x}\|=1 / N, \boldsymbol{x}, \boldsymbol{y} \in \Xi_{N}, \\
0 & \text { otherwise } .\end{cases} \\
\lambda_{N}(\boldsymbol{x}) & =\sum_{\substack{\boldsymbol{y} \in \Xi_{N} \\
\|\boldsymbol{y}-\boldsymbol{x}\|=1 / N}} R_{N}(\boldsymbol{x}, \boldsymbol{y}) .
\end{aligned}
$$

Denote by $D\left(\mathbb{R}_{+}, \Xi_{N}\right)$ the space of right-continuous trajectories $f: \mathbb{R}_{+} \rightarrow \Xi_{N}$ with left-limits, endowed with the Skorohod topology. Let $\mathbf{P}_{\boldsymbol{x}}=\mathbf{P}_{\boldsymbol{x}}^{N}, \boldsymbol{x} \in \Xi_{N}$, be the measure on $D\left(\mathbb{R}_{+}, \Xi_{N}\right)$ induced by the chain $X_{N}(t)$ starting from $\boldsymbol{x}$. Expectation with respect to $\mathbf{P}_{\boldsymbol{x}}$ is denoted by $\mathbf{E}_{\boldsymbol{x}}$.

For a subset $A$ of $\Xi_{N}$, denote by $H_{A}$ (resp. $H_{A}^{+}$) the hitting time of (resp. return time to) the set $A$ :

$$
\begin{aligned}
H_{A} & :=\inf \left\{t>0: X_{N}(t) \in A\right\}, \\
H_{A}^{+} & :=\inf \left\{t>0: X_{N}(t) \in A, X_{N}(s) \neq X_{N}(0) \text { for some } 0<s<t\right\} .
\end{aligned}
$$

The capacity between two disjoint sets $A, B$ of $\Xi_{N}$, denoted by $\operatorname{cap}_{N}(A, B)$, is given by

$$
\operatorname{cap}_{N}(A, B)=\sum_{\boldsymbol{x} \in A} \mu_{N}(\boldsymbol{x}) \lambda_{N}(\boldsymbol{x}) \mathbf{P}_{\boldsymbol{x}}\left[H_{B}<H_{A}^{+}\right]
$$

A. The wells and their capacities. Denote by $\mathfrak{M}$ the set of local minima and by $\mathfrak{S}$ the set of saddle points of $F$ in $\Xi$. Let $\mathfrak{S}_{1}$ be the set of the lowest saddle 
points:

$$
\mathfrak{S}_{1}=\{\boldsymbol{z} \in \mathfrak{S}: F(\boldsymbol{z})=\min \{F(\boldsymbol{y}): \boldsymbol{y} \in \mathfrak{S}\}\} .
$$

We represent by $\boldsymbol{z}^{1,1}, \ldots, \boldsymbol{z}^{1, n_{1}}$ the elements of $\mathfrak{S}_{1}, \mathfrak{S}_{1}=\left\{\boldsymbol{z}^{1,1}, \ldots, \boldsymbol{z}^{1, n_{1}}\right\}$. Starting from $\mathfrak{S}_{1}$, we define inductively a finite sequence of disjoint subsets of $\mathfrak{S}$. Assume that $\mathfrak{S}_{1}, \ldots, \mathfrak{S}_{i}$ have been defined, let $\mathfrak{S}_{i}^{+}=\mathfrak{S}_{1} \cup \cdots \cup \mathfrak{S}_{i}$, and let

$$
\mathfrak{S}_{i+1}=\left\{\boldsymbol{z} \in \mathfrak{S}: F(\boldsymbol{z})=\min \left\{F(\boldsymbol{y}): \boldsymbol{y} \in \mathfrak{S} \backslash \mathfrak{S}_{i}^{+}\right\}\right\} .
$$

We denote by $\boldsymbol{z}^{i, j}, 1 \leq j \leq n_{i}$ the elements of $\mathfrak{S}_{i}$. We obtain in this way a partition $\left\{\mathfrak{S}_{i}: 1 \leq i \leq i_{0}\right\}$ of $\mathfrak{S}$.

We will refer to the index $i$ as the level of a saddle point. Denote by $H_{i}$ the height of the saddle points in $\mathfrak{S}_{i}$ :

$$
H_{i}=F\left(\boldsymbol{z}^{i, 1}\right), \quad 1 \leq i \leq i_{0},
$$

so that $H_{1}<H_{2}<\cdots<H_{i_{0}}$.

For each $1 \leq i \leq i_{0}$, let $\widehat{\Omega}^{i}$ be the subset of $\Xi$ defined by

$$
\widehat{\Omega}^{i}=\left\{\boldsymbol{x} \in \Xi: F(\boldsymbol{x}) \leq F\left(\boldsymbol{z}^{i, 1}\right)\right\} .
$$

By definition, $\widehat{\Omega}^{i} \subset \widehat{\Omega}^{i+1}$. The set $\widehat{\Omega}^{i}$ can be written as a disjoint union of connected components: $\widehat{\Omega}^{i}=\cup_{1 \leq j \leq \varkappa_{i}} \widehat{\Omega}_{j}^{i}$, where $\widehat{\Omega}_{j}^{i} \cap \widehat{\Omega}_{k}^{i}=\varnothing, j \neq k$, and where each set $\widehat{\Omega}_{j}^{i}$ is connected. Some connected component may not contain any saddle point in $\mathfrak{S}_{i}$, and some may contain more than one saddle point. Denote by $\Omega_{j}^{i}, 1 \leq j \leq \ell_{i}$, the connected components $\widehat{\Omega}_{j^{\prime}}^{i}$ which contain a point in $\mathfrak{S}_{i}$, and let $\Omega^{i}=\cup_{1 \leq j \leq \ell_{i}} \Omega_{j}^{i}$. Clearly, the number of components $\Omega_{j}^{i}$ is smaller than the number of elements of $\mathfrak{S}_{i}, \ell_{i} \leq n_{i}$

Each component $\Omega_{j}^{i}$ is a union of wells, $\Omega_{j}^{i}=W_{j, 1}^{i} \cup \cdots \cup W_{j, \ell_{j}^{i}}^{i}$. The sets $W_{j, a}^{i}$ are defined as follows. Let $\Omega_{j}^{i}$ be the interior of $\Omega_{j}^{i}$. Each set $W_{j, a}^{i}$ is the closure of a connected component of $\AA^{i}$. The intersection of two wells is a subset of the set of saddle points: $W_{j, a}^{i} \cap W_{j, b}^{i} \subset \mathfrak{S}_{i}$. Figure 1 illustrates the wells of two connected components of some level. The sets $W_{a}^{\epsilon}$ are introduced just before (2.2).

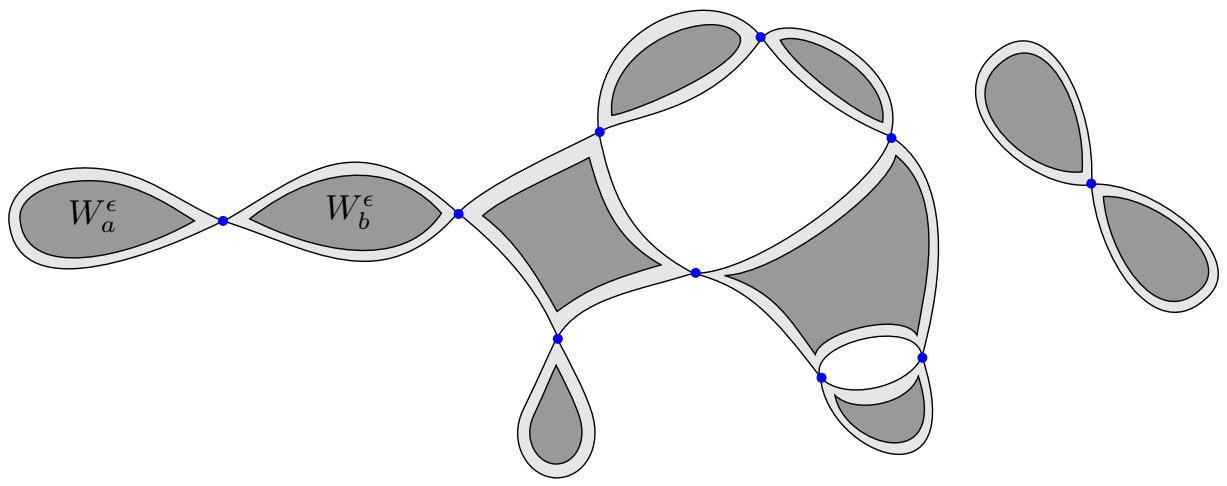

Figure 1. Some wells which form two connected components $\Omega_{1}^{i}$ and $\Omega_{2}^{i}$.

Fix $1 \leq i \leq i_{0}$ and $1 \leq j \leq \ell_{i}$ and a connected component $\Omega=\Omega_{j}^{i}$. To avoid heavy notation, unless when strictly required, we omit from now on the dependence of the sets $\mathfrak{S}_{i}, W_{j, a}^{i}$ and the numbers $\ell_{j}^{i}$ on the indices $i$ and $j$ which are fixed. 
Let $S=\{1, \ldots, \ell\}$ denote the set of the indices of the wells forming the connected component $\Omega$. For $a \neq b \in S$, denote by $\mathfrak{S}_{a, b}$ the set of saddle points separating $W_{a}$ from $W_{b}$,

$$
\mathfrak{S}_{a, b}=\left\{\boldsymbol{z} \in \mathfrak{S}: \boldsymbol{z} \in W_{a} \cap W_{b}\right\},
$$

and denote by $\mathfrak{S}(A), A \subset S$, the set of saddle points separating $\cup_{a \in A} W_{a}$ from $\cup_{a \in A^{c}} W_{a}$ :

$$
\mathfrak{S}(A)=\bigcup_{a \in A, b \in A^{c}} \mathfrak{S}_{a, b} .
$$

For a saddle point $\boldsymbol{z} \in \mathfrak{S}$, denote by $-\mu(\boldsymbol{z})$ the unique negative eigenvalue of the Hessian of $F$ at $\boldsymbol{z}$.

Recall that $F(\boldsymbol{z})=H_{i}, \boldsymbol{z} \in \mathfrak{S}=\mathfrak{S}_{i}$. For $0<\epsilon<H_{i}-H_{i-1}, 1 \leq a \leq \ell$, let $W_{a}^{\epsilon}=\left\{\boldsymbol{x} \in W_{a}: F(\boldsymbol{x})<H_{i}-\epsilon\right\}$, and let

$$
\varepsilon_{N}^{a}=W_{a}^{\epsilon} \cap \Xi_{N}, \quad 1 \leq a \leq \ell, \quad \varepsilon_{N}(A)=\bigcup_{a \in A} \varepsilon_{N}^{a}, A \subset S .
$$

Each well $W_{j, a}^{1}$ contains exactly one local minimum of $F$, while the wells $W_{j, a}^{i}, 1<$ $i \leq i_{0}$, may contain more than one local minimum. Denote by $\left\{\boldsymbol{m}_{a, 1}, \ldots, \boldsymbol{m}_{a, q}\right\}$, $q=q_{a}$, the deepest local minima of $F$ which belong to $W_{j, a}^{i}$ :

$$
\left\{\boldsymbol{m}_{a, 1}, \ldots, \boldsymbol{m}_{a, q}\right\}=\left\{\boldsymbol{y} \in W_{a} \cap \mathfrak{M}: F(\boldsymbol{y})=\min \left\{F\left(\boldsymbol{y}^{\prime}\right): \boldsymbol{y}^{\prime} \in W_{a} \cap \mathfrak{M}\right\}\right\} .
$$

Let $h_{a}=F\left(\boldsymbol{m}_{a, 1}\right)$ and let

$$
\boldsymbol{\mu}(a)=\sum_{k=1}^{q_{a}} \frac{1}{\sqrt{\operatorname{det} \operatorname{Hess} F\left(\boldsymbol{m}_{a, k}\right)}}, a \in S,
$$

where Hess $F(\boldsymbol{x})$ represents the Hessian of $F$ calculated at $\boldsymbol{x}$, and $\operatorname{det} \operatorname{Hess} F(\boldsymbol{x})$ its determinant. A calculation, presented in 6.5, shows that for each $a \in S$,

$$
\mu_{N}\left(\varepsilon_{N}^{a}\right)=\left[1+o_{N}(1)\right] \frac{(2 \pi N)^{d / 2}}{Z_{N}} e^{-N h_{a}} \boldsymbol{\mu}(a) .
$$

The next result and Theorem 2.2 below are discrete versions of a result of Bovier, Eckhoff, Gayrard and Klein [9]. The proofs are based on the proof of Theorem 3.1 in [9] and on [5, 6].

Theorem 2.1. For every proper subset $A$ of $S$,

$$
\lim _{N \rightarrow \infty} \frac{Z_{N}}{(2 \pi N)^{d / 2}} 2 \pi N e^{N H_{i}} \operatorname{cap}_{N}\left(\mathcal{E}_{N}(A), \mathcal{E}_{N}\left(A^{c}\right)\right)=\sum_{\boldsymbol{z} \in \mathfrak{S}(A)} \frac{\mu(\boldsymbol{z})}{\sqrt{-\operatorname{det} \operatorname{Hess} F(\boldsymbol{z})}} .
$$

This result together with two other estimates permit to prove the metastable behavior of the Markov chain $X_{N}(t)$ among the shallowest valleys $\mathcal{E}_{N}^{a}$. To examine the metastable behavior of the chain $X_{N}(t)$ on deeper wells we need to extend Theorem 2.1 to disjoint sets $A, B$ which do not form a partition of $S, A \cup B \neq S$. The statement of this extension and its proof requires the introduction of a graph.

B. A Graph associated to the chain. Let $\mathbb{G}=(S, E)$ be the weighted graph whose vertices are $S=\{1, \ldots, \ell\}$, the indices of the sets $W_{a}$. Place an edge between $a$ and $b \in S$ if and only if there exists a saddle point $\boldsymbol{z}$ belonging to $W_{a} \cap W_{b}$, i.e., 
if $\mathfrak{S}_{a, b} \neq \varnothing$. The weight of the edge between $a$ and $b$, denoted by $\boldsymbol{c}(a, b)$, is set to be

$$
\boldsymbol{c}(a, b)=\sum_{\boldsymbol{z} \in \mathfrak{S}_{a, b}} \frac{\mu(\boldsymbol{z})}{\sqrt{-\operatorname{det} \operatorname{Hess} F(\boldsymbol{z})}} .
$$

Note that $\boldsymbol{c}(a, b)$ vanishes if there is no saddle point $\boldsymbol{z}$ belonging to $W_{a} \cap W_{b}$ and that the weights are independent of $N$. Figure 2 present the weighted graph associated to one of the connected component of Figure 1.
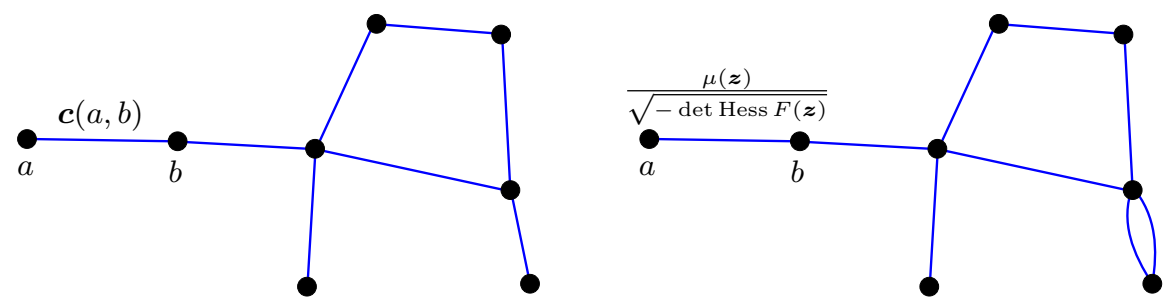

Figure 2. The simple weighted graph and the graph with multiple edges associated to one of the connected components of Figure 1

The graph $\mathbb{G}$ has to be interpreted as an electrical network, where the weights $\boldsymbol{c}(a, b)$ represent the conductances. It would be more natural to start with a graph with multiple edges, each edge corresponding to a saddle point $\boldsymbol{z}$. However, adding the parallel conductances one can reduce the graph with multiple edges to the above graph.

Let

$$
c_{N}(a, b)=\frac{(2 \pi N)^{d / 2}}{Z_{N}} \frac{e^{-N H_{i}}}{2 \pi N} \boldsymbol{c}(a, b), a, b \in S .
$$

It follows from Theorem 2.1 and from a calculation that

$$
\begin{aligned}
& c_{N}(a, b)= \\
& \quad\left[1+o_{N}(1)\right] \frac{1}{2}\left\{\operatorname{cap}_{N}\left(\mathcal{E}_{N}^{a}, \breve{\mathcal{E}}_{N}^{a}\right)+\operatorname{cap}_{N}\left(\mathcal{E}_{N}^{b}, \breve{\mathcal{E}}_{N}^{b}\right)-\operatorname{cap}_{N}\left(\mathcal{E}_{N}^{a} \cup \mathcal{E}_{N}^{b}, \cup_{c \neq a, b} \mathcal{E}_{N}^{c}\right)\right\},
\end{aligned}
$$

where, $\breve{\mathcal{E}}_{N}^{a}=\cup_{c \neq a} \mathcal{E}_{N}^{c}$. This explains the definition of $c_{N}(a, b)$. Moreover, by [2, Lemma 6.8], $c_{N}(a, b)$ is equal to $\mu_{N}\left(\mathcal{E}_{N}^{a}\right) r_{N}\left(\mathcal{E}_{N}^{a}, \mathcal{E}_{N}^{b}\right)$, where $r_{N}=r_{N}^{1}$ represents the average rates introduced below in 6.1).

For two disjoint subsets $A, B$ of $S$, denote by $\operatorname{cap}_{\mathbb{G}}(A, B)$ the conductance between $A$ and $B$. To define the conductance, denote by $\left\{Y_{k}: k \geq 0\right\}$ the discrete-time random walk on $S$ which jumps from $a$ to $b$ with probability

$$
p(a, b)=\frac{c(a, b)}{\sum_{b^{\prime} \in S} \boldsymbol{c}\left(a, b^{\prime}\right)} .
$$

Denote by $\mathbb{P}_{a}^{Y}, a \in S$, the distribution of the chain $Y_{k}$ starting from $a$ and by $V_{A, B}$, $A, B \subset S, A \cap B=\varnothing$, the equilibrium potential between $A$ and $B$ :

$$
V_{A, B}(b)=\mathbb{P}_{b}^{Y}\left[H_{A}<H_{B}\right], \quad b \in S,
$$


where $H_{C}, C \subset S$, represents the hitting time of $C: H_{C}=\min \left\{k \geq 0: Y_{k} \in C\right\}$. The conductance between $A$ and $B$ is defined as

$$
\operatorname{cap}_{\mathbb{G}}(A, B)=\frac{1}{2} \sum_{a, b \in S} \boldsymbol{c}(a, b)\left[V_{A, B}(b)-V_{A, B}(a)\right]^{2} .
$$

By [16. Proposition 3.1.2] the conductance between $A$ and $B$ coincides with the capacity between $A$ and $B$. The next result establishes that the capacities for the chain $X_{N}(t)$ can be computed from the conductances on the finite graph $\mathbb{G}$.

Theorem 2.2. For every disjoint subsets $A, B$ of $S$,

$$
\operatorname{cap}_{N}\left(\mathcal{E}_{N}(A), \mathcal{E}_{N}(B)\right)=\left[1+o_{N}(1)\right] \frac{(2 \pi N)^{d / 2}}{Z_{N}} \frac{e^{-N H_{i}}}{2 \pi N} \operatorname{cap}_{\mathbb{G}}(A, B) .
$$

Remark 2.3. It follows from the proofs of Theorems 2.1 and 2.2 that both statements remain in force if we replace the sets $\mathcal{E}_{N}^{a}$ by singletons $\left\{\boldsymbol{x}_{N}^{a}\right\}$, where $\boldsymbol{x}_{N}^{a} \in \mathcal{E}_{N}^{a}$. In this case the sets $\mathcal{E}_{N}(A)$ become $\left\{\boldsymbol{x}_{N}^{a}: a \in A\right\}$.

C. Metastability. The Markov chain $X_{N}(t)$ exhibits a metastable behavior among the wells of each connected component $\Omega_{j}^{i}$. The description of this behavior requires some further notation.

Recall that $h_{a}=F\left(\boldsymbol{m}_{a, 1}\right)$ represents the value of $F$ at a deepest minima of the well $W_{a}$. Let $\hat{\theta}_{a}=H_{i}-h_{a}>0, a \in S$, be the depth of the well $W_{a}$. The depths $\hat{\theta}_{a}$ provide the time-scale at which a metastable behavior is observed. Let $\theta_{1}<\theta_{2}<\cdots<\theta_{n}, n \leq \ell$, be the increasing enumeration of the sequence $\hat{\theta}_{a}$, $1 \leq a \leq \ell$ :

$$
\left\{\hat{\theta}_{1}, \ldots, \hat{\theta}_{\ell}\right\}=\left\{\theta_{1}, \ldots, \theta_{n}\right\} .
$$

Of course, $n$ and $\theta_{m}$ depend on the component $\Omega_{j}^{i}$. If we need to stress this dependence, we will denote $n, \theta_{m}$ by $n_{i, j}, \theta_{m}^{i, j}$, respectively.

The chain exhibits a metastable behavior on $n$ different time scales in the set $\Omega$. Let $T_{m}=\left\{a \in S: \hat{\theta}_{a}=\theta_{m}\right\}, 1 \leq m \leq n$, so that $T_{1}, \ldots, T_{n}$ forms a partition of $S$, and let

$$
S_{m}=T_{m} \cup \cdots \cup T_{n}, \quad 1 \leq m \leq n .
$$

Define the projection $\Psi_{N}^{m}: \Xi_{N} \rightarrow S_{m} \cup\{N\}, 1 \leq m \leq n$, as

$$
\Psi_{N}^{m}(\boldsymbol{x})=\sum_{a \in S_{m}} a \mathbf{1}\left\{\boldsymbol{x} \in \mathcal{E}_{N}^{a}\right\}+N \mathbf{1}\left\{\boldsymbol{x} \notin \bigcup_{a \in S_{m}} \varepsilon_{N}^{a}\right\} .
$$

Denote by $\boldsymbol{X}_{N}^{m}(t)$ the projection of the Markov chain $X_{N}(t)$ by $\Psi_{N}^{m}$ :

$$
\boldsymbol{X}_{N}^{m}(t)=\Psi_{N}^{m}\left(X_{N}(t)\right) .
$$

Fix $1 \leq m \leq n$. We introduce some notation to define the asymptotic dynamics of the process $\boldsymbol{X}_{N}^{m}(t)$. The time scale in which the process $\boldsymbol{X}_{N}^{m}$ evolves, denoted by $\beta_{m}=\beta_{m}(N)$, is given by

$$
\beta_{m}=2 \pi N e^{\theta_{m} N} .
$$

For $a, b$ in $S_{m}$, let

$$
\boldsymbol{c}_{m}(a, b)=\frac{1}{2}\left\{\operatorname{cap}_{\mathbb{G}}\left(\{a\}, S_{m} \backslash\{a\}\right)+\operatorname{cap}_{\mathbb{G}}\left(\{b\}, S_{m} \backslash\{b\}\right)-\operatorname{cap}_{\mathbb{G}}\left(\{a, b\}, S_{m} \backslash\{a, b\}\right)\right\} .
$$


Note that $\boldsymbol{c}_{m}(a, b)$ represents the conductance between $a$ and $b$ for the electrical circuit obtained from $\mathbb{G}$ by removing the vertices in $S_{m}^{c}$. In particular, $\boldsymbol{c}_{1}(a, b)=$ $\boldsymbol{c}(a, b)$ for $a, b \in S_{m}$. Let

$$
\boldsymbol{r}_{m}(a, b)= \begin{cases}\boldsymbol{c}_{m}(a, b) / \boldsymbol{\mu}(a) & a \in T_{m}, b \in S_{m}, \\ 0 & a \in S_{m+1}, b \in S_{m} .\end{cases}
$$

Recall from [18 the definition of the soft topology.

Theorem 2.4. Fix $1 \leq i \leq i_{0}, 1 \leq j \leq \ell_{i}, 1 \leq m \leq n_{i, j}, a \in S_{m}$ and $a$ sequence of configurations $\boldsymbol{x}_{N}$ in $\mathcal{E}_{N}^{a}$. Under $\mathbf{P}_{\boldsymbol{x}_{N}}$, the time re-scaled projection $\mathbb{X}_{N}^{m}(t)=\boldsymbol{X}_{N}^{m}\left(t \beta_{m}\right)$ converges in the soft topology to a $S_{m}$-valued continuous-time Markov chain $\mathbb{X}^{m}(t)$ whose jump rates are given by 2.9. In particular, the points in $S_{m+1}$ are absorbing for the chain $\mathbb{X}^{m}(t)$.

Remark 2.5. Theorem 2.4 states that the weighted graph $\mathbb{G}$, the measure $\boldsymbol{\mu}$ and the sequence $\beta_{m}(N)$ describe the evolution of the chain $X_{N}(t)$ in the connected component $\Omega$. The weighted graph with multiple edges would describe more accurately the chain $X_{N}(t)$, providing the probability that the chain leaves a well $W_{a}$ through a mesoscopic neighborhood of a saddle point $\boldsymbol{z} \in \mathfrak{S}$. This statement is made precise in Theorem 2.7 below.

Remark 2.6. Nothing prevent two time-scales at different levels to be equal, or two time scales in different connected components of the same level to be equal. It is possible that $\theta_{m}^{i, j}=\theta_{m^{\prime}}^{i^{\prime}, j^{\prime}}$ for some $i \neq i^{\prime}$ or that $\theta_{m}^{i, j}=\theta_{m^{\prime}}^{i, j^{\prime}}$ for some $j \neq j^{\prime}$.

D. Exit points from a well. Fix $1 \leq i \leq i_{0}, 1 \leq j \leq \ell_{i}$, and recall that we denote by $W_{a}=W_{j, a}^{i}, a \in S=\left\{1, \ldots, \ell_{j}^{i}\right\}$, the wells which form the connected component $\Omega_{j}^{i}$. The last result of this article states that the chain $X_{N}(t)$ leaves the set $W_{a}$ through a neighborhood of a saddle point $\boldsymbol{z}$ in the boundary of $W_{a}$ with probability $\omega(\boldsymbol{z}) / \sum_{\boldsymbol{z}^{\prime}} \omega\left(\boldsymbol{z}^{\prime}\right)$, where the summation is carried over all saddle points in the boundary of $W_{a}$ and where

$$
\omega(\boldsymbol{z})=\frac{\mu(\boldsymbol{z})}{\sqrt{-\operatorname{det} \operatorname{Hess} F(\boldsymbol{z})}} \cdot
$$

Let $\delta_{N}$ be a sequence such that $\delta_{N} \ll N^{-3 / 4}, N^{d+1} \exp \left\{-N \delta_{N}\right\} \rightarrow 0$. Denote by $\Omega_{N}=\Omega_{j, N}^{i}$ the connected component of the set $\left\{\boldsymbol{x} \in \Xi: F(\boldsymbol{x}) \leq H_{i}+\delta_{N}\right\}$ which contains $\Omega_{j}^{i}$. Since $\delta_{N} \downarrow 0$, for $N$ large enough, $\Omega_{j, N}^{i} \cap \Omega_{j^{\prime}, N}^{i}=\varnothing$ for all $j^{\prime} \neq j$. In particular, for $N$ large enough there is a one-to-one correspondance between $\Omega_{j}^{i}$ and $\Omega_{j, N}^{i}$.

Fix $a \in S$ and let $\mathfrak{S}_{a}$ be the set of saddle points in the boundary of $W_{a}, \mathfrak{S}_{a}=$ $\cup_{b \in S, b \neq a} \mathfrak{S}_{a, b}$. Denote by $\partial \Omega_{N}$ the boundary of $\Omega_{N}$ and by $B_{\epsilon}(\boldsymbol{x})$ the open ball of radius $\epsilon>0$ around $\boldsymbol{x} \in \Xi$. We modify the set $\partial \Omega_{N}$ around each saddle point $z \in \mathfrak{S}_{a}$ to obtain a closed manifold $D_{a} \subset \Omega_{N}$.

Fix a saddle point $\boldsymbol{z} \in \mathfrak{S}_{a}$ and recall condition (H3) on $F$. Denote by $-\mu<$ $0<\lambda_{2} \leq \cdots \leq \lambda_{d}$ the eigenvalues of $\operatorname{Hess} F(\boldsymbol{z})$, and by $\boldsymbol{v}, \boldsymbol{w}^{i}, 2 \leq i \leq d$, an associated orthonormal basis of eigenvectors. Let $\mathbb{H}=\mathbb{H}_{\boldsymbol{z}}$ be the $(d-1)$-dimensional hyperplane generated by the vectors $\boldsymbol{w}^{i}, 2 \leq i \leq d$. By a Taylor expansion, there exists $\epsilon>0$ such that

$$
F(\boldsymbol{x}) \geq H_{i}+\frac{\lambda_{2}}{4}\|\boldsymbol{x}-\boldsymbol{z}\|^{2}
$$


for $\boldsymbol{x} \in \boldsymbol{z}+\mathbb{H}=\{\boldsymbol{z}+\boldsymbol{y}: \boldsymbol{y} \in \mathbb{H}\}$ such that $\|\boldsymbol{x}-\boldsymbol{z}\| \leq \epsilon$. Let

$$
D_{\boldsymbol{z}}=\left\{\boldsymbol{y} \in(\boldsymbol{z}+\mathbb{H}) \cap B_{\epsilon}(\boldsymbol{z}): F(\boldsymbol{y}) \leq H_{i}+\delta_{N}\right\} .
$$

We intersected the set $\boldsymbol{z}+\mathbb{H}$ with the set $B_{\epsilon}(\boldsymbol{z})$ to avoid including in $D_{\boldsymbol{z}}$ points which are far from $\boldsymbol{z}$.

The set $D_{a}=D_{a}^{N}$ is defined as follows. For each $\boldsymbol{z} \in \mathfrak{S}_{a}$, remove from $\partial \Omega_{N}$ the set $(\boldsymbol{z}+\mathbb{H}) \cap \partial \Omega_{N} \cap B_{\epsilon}(\boldsymbol{z})$. As before, the set $B_{\epsilon}(\boldsymbol{z})$ has been introduced to avoid removing from $\partial \Omega_{N}$ points which are far from $z$. Denote by $\Omega_{N}^{1}$ the set obtained after this operation, which is a finite union of connected sets. Remove from $\Omega_{N}^{1}$ all connected component which contain a point close to some saddle point which does not belong to $\mathfrak{S}_{a}$. Denote this new set by $\Omega_{N}^{2}$. $D_{a}$ is the union of $\Omega_{N}^{2}$ with all set $D_{\boldsymbol{z}}, \boldsymbol{z} \in \mathfrak{S}_{a}$ :

$$
D_{a}=\bigcup_{\boldsymbol{z} \in \mathfrak{S}_{a}} D_{\boldsymbol{z}} \cup \Omega_{N}^{2}
$$

Let $\mathcal{D}_{a}, \mathcal{D}_{\boldsymbol{z}} \subset \Xi_{N}$, be discretizations of the sets $D_{a}, D_{\boldsymbol{z}}: \mathcal{D}_{a}=\left\{\boldsymbol{x} \in \Xi_{N}\right.$ : $\left.d\left(\boldsymbol{x}, D_{a}\right) \leq 1 / N\right\}$, where $d$ stands for the Euclidean distance, $d(\boldsymbol{x}, A)=\inf _{\boldsymbol{y} \in A} \| \boldsymbol{x}-$ $\boldsymbol{y} \|$.

Theorem 2.7. Fix $1 \leq i \leq i_{0}, 1 \leq j \leq \ell_{i}$, and $a \in S=\left\{1, \ldots \ell_{j}^{i}\right\}$. Let . For all $\boldsymbol{z} \in \mathfrak{S}_{a}$, and all sequences $\left\{\boldsymbol{x}_{N}: N \geq 1\right\}, \boldsymbol{x}_{N} \in \mathcal{E}_{N}^{a}$,

$$
\lim _{N \rightarrow \infty} \mathbf{P}_{\boldsymbol{x}_{N}}\left[H_{\mathcal{D}_{a}}=H_{\mathcal{D}_{\boldsymbol{z}}}\right]=\frac{\omega(\boldsymbol{z})}{\sum_{\boldsymbol{z}^{\prime} \in \mathfrak{S}_{a}} \omega\left(\boldsymbol{z}^{\prime}\right)} .
$$

The proof of Lemma 7.1 yields the last result.

Proposition 2.8. Let $D \subset \Xi$ be a domain with a smooth boundary, and let $m=: \inf _{\boldsymbol{y} \in \partial D} F(\boldsymbol{y})$. Fix a sequence $\left\{\epsilon_{N}: N \geq 1\right\}$ of positive numbers such that $\lim _{N} N^{d+1} \exp \left\{-N \epsilon_{N}\right\}=0$, and let $D_{N}=\Xi_{N} \cap D, B_{N}=\left\{\boldsymbol{x} \in \partial D_{N}: F(\boldsymbol{x}) \leq\right.$ $\left.m+2 \epsilon_{N}\right\}$. Fix a point $\boldsymbol{x} \in D$ such that $F(\boldsymbol{x})<m$ and for which there exists a continuous path $\boldsymbol{x}(t), 0 \leq t \leq 1$, from $B_{N}$ to $\boldsymbol{x}$ such that $F(\boldsymbol{x}(t)) \leq m+\epsilon_{N}$ for all $0 \leq t \leq 1$. Then,

$$
\lim _{N \rightarrow \infty} \mathbf{P}_{\boldsymbol{x}_{N}}\left[H_{\partial D_{N}}=H_{B_{N}}\right]=1
$$

where $\boldsymbol{x}_{N} \in D_{N},\left\|\boldsymbol{x}_{N}-\boldsymbol{x}\right\| \leq 1 / N$.

We conclude this section with some comments. Bianchi, Bovier and Ioffe [5, 6] examined the metastable behavior of the Curie-Weiss model with random external fields. In this case the potential $F$ becomes a sequence of potentials $F_{N}$ which converges to some function $F_{\infty}$. The authors assumed that the parameter of the model, the distribution of the external field, were chosen to guarantee that all wells do not have saddle points at the same height. In this case, the metastable behavior of the chain consists in staying for an exponential time in some well and then to jump to a deeper well in which the chain remains trapped for ever.

To observe a metastable behavior similar to the one described in Theorem 2.4. one has to tune the distribution of the external field in a way that the wells associated to $F_{\infty}$ have more than one saddle point at the same height. In this case, however, the metastable behavior might depend on the subsequence of $N$.

To illustrate this possibility, consider the following one-dimensional example. Let $F_{N}$ be a sequence of potentials which converge uniformly to a potential $F_{\infty}$. Fix two local maxima of $F_{\infty}$, supposed to be at the same height, $F_{\infty}(\boldsymbol{z})=F_{\infty}\left(\boldsymbol{z}^{\prime}\right)$, and assume that the interval $\left(\boldsymbol{z}, \boldsymbol{z}^{\prime}\right)$ is a well, $F_{\infty}(\boldsymbol{x})<F_{\infty}(\boldsymbol{z})$ for $\boldsymbol{z}<\boldsymbol{x}<\boldsymbol{z}^{\prime}$. Suppose 
also that $F_{N}$ has two local maxima $\boldsymbol{z}_{N}, \boldsymbol{z}_{N}^{\prime}$ such that $\boldsymbol{z}_{N} \rightarrow \boldsymbol{z}, \boldsymbol{z}_{N}^{\prime} \rightarrow \boldsymbol{z}^{\prime}$, that $\left(\boldsymbol{z}_{N}, \boldsymbol{z}_{N}^{\prime}\right)$ is a well for $F_{N}$, and that there exists subsequences $N^{\prime}$ and $N^{\prime \prime}$ such that

$$
N^{\prime}\left[F_{N^{\prime}}\left(\boldsymbol{z}_{N^{\prime}}^{\prime}\right)-F_{N^{\prime}}\left(\boldsymbol{z}_{N^{\prime}}\right)\right] \leq-\epsilon, \quad N^{\prime \prime}\left[F_{N^{\prime \prime}}\left(\boldsymbol{z}_{N^{\prime \prime}}^{\prime}\right)-F_{N^{\prime \prime}}\left(\boldsymbol{z}_{N^{\prime \prime}}\right)\right] \geq \epsilon
$$

for some $\epsilon>0$. In this case, in view of the results presented in this section, starting from a local minima in $\left(\boldsymbol{z}_{N}, \boldsymbol{z}_{N}^{\prime}\right)$, along the subsequence $N^{\prime}$, almost surely the chain will escape from $\left(\boldsymbol{z}_{N}, \boldsymbol{z}_{N}^{\prime}\right)$ through a neighborhood of $\boldsymbol{z}_{N}^{\prime}$, while along the subsequence $N^{\prime \prime}$ almost surely it will escape from $\left(\boldsymbol{z}_{N}, \boldsymbol{z}_{N}^{\prime}\right)$ through a neighborhood of $\boldsymbol{z}_{N}$.

This is what happens for the Curie-Weiss model with an external field, random or not, if there exist saddle points at the same height. For the metastable behavior not to depend on particular subsequences, one needs to impose some strong conditions on the asymptotic behavior of the sequence $F_{N}$.

The article is divided as follows. In Section 3 we prove the upper bound for the capacities appearing in the statement of Theorem 2.1 and in Section 4 the lower bound. In Section 5 we prove Theorem 2.2, in Section 6, Theorem 2.4, and in Section 7, Theorem 2.7.

\section{UPPER BOUND FOR THE CAPACITIES}

We prove in this section the upper bound of Theorem 2.1. The proof is based on ideas of [9, 5, 6] and on the Dirichlet principle [16, Proposition 3.1.3] which expresses the capacity between two sets as an infimum of the Dirichlet form: for two disjoint subsets $A, B$ of $\Xi_{N}$,

$$
\operatorname{cap}_{N}(A, B)=\inf _{f} D_{N}(f)
$$

where the infimum is carried over all functions $f: \Xi_{N} \rightarrow \mathbb{R}$ such that $f(\boldsymbol{x})=1$, $\boldsymbol{x} \in A, f(\boldsymbol{y})=0, \boldsymbol{y} \in B$, and where $D_{N}(f)$ stands for the Dirichlet form of $f$,

$D_{N}(f)=\sum_{\boldsymbol{x} \in \Xi_{N}} f(\boldsymbol{x})\left(-L_{N} f\right)(\boldsymbol{x}) \mu_{N}(\boldsymbol{x})=\frac{1}{2} \sum_{\boldsymbol{x}, \boldsymbol{y} \in \Xi_{N}} \mu_{N}(\boldsymbol{x}) R_{N}(\boldsymbol{x}, \boldsymbol{y})[f(\boldsymbol{y})-f(\boldsymbol{x})]^{2}$.

Proposition 3.1. For every proper subset $A$ of $\{1, \ldots, \ell\}$,

$\limsup _{N \rightarrow \infty} \frac{Z_{N}}{(2 \pi N)^{d / 2}} 2 \pi N e^{N F(\boldsymbol{z})} \operatorname{cap}_{N}\left(\mathcal{E}_{N}(A), \mathcal{E}_{N}\left(A^{c}\right)\right) \leq \sum_{\boldsymbol{z} \in \mathfrak{S}(A)} \frac{\mu(\boldsymbol{z})}{\sqrt{-\operatorname{det} \operatorname{Hess} F(\boldsymbol{z})}}$.

The proof of this proposition is divided in several lemmas. The main point is that the capacities depend on the behavior of the function $F$ around the saddle points of $F$.

Fix a saddle point $\boldsymbol{z}$ of $F$ and denote by $\mathbb{M}=(\operatorname{Hess} F)(\boldsymbol{z})$ the Hessian of $F$ at $\boldsymbol{z}$. Denote by $-\mu$ the negative eigenvalue of $\mathbb{M}$ and by $0<\lambda_{2} \leq \cdots \leq \lambda_{d}$ the positive eigenvalues. Let $\boldsymbol{v}, \boldsymbol{w}^{i}, 2 \leq i \leq d$, be orthonormal eigenvectors associated to the eigenvalues $-\mu, \lambda_{i}$, respectively. We sometimes denote $\boldsymbol{v}$ by $\boldsymbol{w}^{1}$ and $-\mu$ by $\lambda_{1}$.

Let $\mathbb{V}$ the $(d \times d)$-matrix whose $j$-th column is the vector $\boldsymbol{w}^{j}$ and denote by $\mathbb{V}^{*}$ its transposition. Denote by $\mathbb{D}$ the diagonal matrix whose diagonal entries are $\lambda_{i}$ so that $\mathbb{M}=\mathbb{V} \mathbb{D} \mathbb{V}^{*}$. Let $\mathbb{D}_{\star}$ be the matrix $\mathbb{D}$ in which we replaced the negative eigenvalue $\lambda_{1}$ by its absolute value $\mu$ and let

$$
\mathbb{M}_{\star}=\mathbb{V} \mathbb{D}_{\star} \mathbb{V}^{*} .
$$

Clearly, $\operatorname{det} \mathbb{M}=-\operatorname{det} \mathbb{M}_{\star}$. 
Let $B_{N}=B_{N}^{z}=\mathcal{B}^{z} \cap \Xi_{N}$ be a mesoscopic neighborhood of $\boldsymbol{z}$ :

$$
\mathcal{B}^{\boldsymbol{z}}=\left\{\boldsymbol{x} \in \Xi:|(\boldsymbol{x}-\boldsymbol{z}) \cdot \boldsymbol{v}| \leq \varepsilon_{N}, \max _{2 \leq j \leq d}\left|(\boldsymbol{x}-\boldsymbol{z}) \cdot \boldsymbol{w}^{j}\right| \leq 2 \sqrt{\mu / \lambda_{j}} \varepsilon_{N}\right\},
$$

where $N^{-1} \ll \varepsilon_{N} \ll 1$ is a sequence of positive numbers to be chosen later. Unless needed, we omit the index $\boldsymbol{z}$ from the notation $B_{N}^{\boldsymbol{z}}$. Denote by $\partial B_{N}$ the outer boundary of $B_{N}$ defined by

$$
\partial B_{N}=\left\{\boldsymbol{x} \in \Xi_{N} \backslash B_{N}: \exists \boldsymbol{y} \in B_{N} \text { s.t. }\|\boldsymbol{y}-\boldsymbol{x}\|=N^{-1}\right\},
$$

and let $\partial_{-} B_{N}, \partial_{+} B_{N}$ be the pieces of the outer boundary of $B_{N}$ defined by

$$
\begin{gathered}
\partial_{-} B_{N}=\left\{\boldsymbol{x} \in \partial B_{N}:(\boldsymbol{x}-\boldsymbol{z}) \cdot \boldsymbol{v}<-\varepsilon_{N}\right\}, \\
\partial_{+} B_{N}=\left\{\boldsymbol{x} \in \partial B_{N}:(\boldsymbol{x}-\boldsymbol{z}) \cdot \boldsymbol{v}>\varepsilon_{N}\right\} .
\end{gathered}
$$

The Dirichlet forms in the sets $B_{N}$. Denote by $D_{N}\left(f ; B_{N}\right)$ the piece of the Dirichlet form of a function $f: \Xi_{N} \rightarrow \mathbb{R}$ corresponding to the edges in the set $B_{N}$ :

$$
D_{N}\left(f ; B_{N}\right)=\sum_{i=1}^{d} \sum_{\boldsymbol{x} \in B_{N}} \mu_{N}(\boldsymbol{x}) R_{N}\left(\boldsymbol{x}, \boldsymbol{x}+\boldsymbol{e}_{i}\right)\left[f\left(\boldsymbol{x}+\boldsymbol{e}_{i}\right)-f(\boldsymbol{x})\right]^{2},
$$

where $\left\{\mathfrak{e}_{1}, \ldots, \mathfrak{e}_{d}\right\}$ is the canonical basis of $\mathbb{R}^{d}$ and $\boldsymbol{e}_{i}=N^{-1} \mathfrak{e}_{i}$.

Let $\mu_{N}^{z}$ be the measure on $B_{N}$ given by

$$
\mu_{N}^{\boldsymbol{z}}(\boldsymbol{x})=\frac{1}{Z_{N}} e^{-N F(\boldsymbol{z})} e^{-(1 / 2) N(\boldsymbol{y} \cdot \mathbb{M} \boldsymbol{y})},
$$

where $\boldsymbol{y}=\boldsymbol{x}-\boldsymbol{z}$, and where $\boldsymbol{v} \cdot \boldsymbol{w}$ represents the scalar product between $\boldsymbol{v}$ and $\boldsymbol{w}$. Denote by $D_{N}^{z}$ the Dirichlet form defined by

$$
D_{N}^{\boldsymbol{z}}(f)=\sum_{i=1}^{d} \sum_{\boldsymbol{x} \in B_{N}} \mu_{N}^{\boldsymbol{z}}(\boldsymbol{x})\left[f\left(\boldsymbol{x}+\boldsymbol{e}_{i}\right)-f(\boldsymbol{x})\right]^{2} .
$$

The next assertion follows from an elementary computation and from assumption (H1).

Assertion 3.A. For every function $f: \Xi_{N} \rightarrow \mathbb{R}$,

$$
D_{N}\left(f ; B_{N}\right)=\left[1+O\left(\varepsilon_{N}\right)+O\left(N \varepsilon_{N}^{3}\right)\right] D_{N}^{z}(f) .
$$

The equilibrium potential. We introduce in this subsection an approximation in the set $B_{N}$ of the solution to the Dirichlet variational problem for the capacity. To explain the choice, consider a one-dimensional random walk on the interval $I_{N}=\left\{-K_{N} / N, \ldots,\left(K_{N}-1\right) / N, K_{N} / N\right\}$ whose Dirichlet form $D_{N}$ is given by

$$
D_{N}(f)=\sum_{k} e^{\mu N k^{2}}\left[f\left(k+N^{-1}\right)-f(k)\right]^{2},
$$

where the sum is performed over $k \in I_{N}, k \neq K_{N} / N$. An elementary computation shows that the equilibrium potential $V(k / N)=P_{k / N}\left[H_{K_{N} / N}<H_{-K_{N} / N}\right]$ is given by

$$
V(k / N)=\frac{\sum_{j=-K_{N} / N}^{(k-1) / N} e^{-\mu N j^{2}}}{\sum_{j=-K_{N} / N}^{\left(K_{N}-1\right) / N} e^{-\mu N j^{2}}} \sim \frac{\int_{-\infty}^{k / N} e^{-\mu N r^{2}} d r}{\int_{-\infty}^{\infty} e^{-\mu N r^{2}} d r},
$$

where the last approximation holds provided $\sqrt{N} \ll K_{N} \ll N$. 
In view of the previous observation, let $f: \mathbb{R} \rightarrow \mathbb{R}_{+}$be given by

$$
f_{N}(r)=\frac{\int_{-\infty}^{r} e^{-(1 / 2) N \mu s^{2}} d s}{\int_{-\infty}^{\infty} e^{-(1 / 2) N \mu s^{2}} d s}=\sqrt{\frac{N \mu}{2 \pi}} \int_{-\infty}^{r} e^{-(1 / 2) N \mu s^{2}} d s .
$$

The function $V_{N}$ defined below is an approximation on the set $B_{N}$ for the equilibrium potential between $\partial_{-} B_{N}$ and $\partial_{+} B_{N}$ :

$$
V_{N}(\boldsymbol{x})=V_{N}^{\boldsymbol{z}}(\boldsymbol{x})=f_{N}([\boldsymbol{x}-\boldsymbol{z}] \cdot \boldsymbol{v}) .
$$

Assertion 3.B. Assume that $N^{-1 / 2} \ll \varepsilon_{N} \ll N^{-1 / 3}$. Then,

$$
\frac{Z_{N}}{(2 \pi N)^{d / 2}} 2 \pi N e^{N F(\boldsymbol{z})} D_{N}\left(V_{N} ; B_{N}\right)=\left[1+o_{N}(1)\right] \frac{\mu}{\sqrt{-\operatorname{det} \operatorname{Hess} F(\boldsymbol{z})}} \cdot
$$

Proof. By Assertion 3.A, it is enough to estimate $D_{N}^{z}\left(V_{N}\right)$. By definition of the Dirichlet form $D_{N}^{z}$,

$$
Z_{N} e^{N F(\boldsymbol{z})} D_{N}^{\boldsymbol{z}}\left(V_{N}\right)=\sum_{i=1}^{d} \sum_{\boldsymbol{x} \in B_{N}} e^{-(1 / 2) N(\boldsymbol{y} \cdot \mathbb{M} \boldsymbol{y})}\left[V_{N}\left(\boldsymbol{x}+\boldsymbol{e}_{i}\right)-V_{N}(\boldsymbol{x})\right]^{2},
$$

where $\boldsymbol{y}=\boldsymbol{x}-\boldsymbol{z}$. Denote by $\boldsymbol{v}_{1}, \ldots, \boldsymbol{v}_{d}$ the coordinates of the vector $\boldsymbol{v}$ and recall that $\|\boldsymbol{v}\|=1$. Recall the definition of the matrix $\mathbb{M}_{\star}$ introduced in (3.1). Since $(\boldsymbol{y} \cdot \mathbb{M} \boldsymbol{y})=\sum_{1 \leq j \leq d} \lambda_{j}\left(\boldsymbol{y} \cdot \boldsymbol{w}^{j}\right)^{2}$, by definition of $V_{N}$ this sum is equal to

$$
\begin{aligned}
{[1} & \left.+o_{N}(1)\right] \frac{\mu}{2 \pi N} \sum_{i=1}^{d} \boldsymbol{v}_{i}^{2} \sum_{\boldsymbol{x} \in B_{N}} e^{-(1 / 2) N(\boldsymbol{y} \cdot \mathbb{M} \boldsymbol{y})} e^{-\mu N(\boldsymbol{y} \cdot \boldsymbol{v})^{2}} \\
& =\left[1+o_{N}(1)\right] \frac{\mu}{2 \pi N} \sum_{\boldsymbol{x} \in B_{N}} e^{-(1 / 2) N\left(\boldsymbol{y} \cdot \mathbb{M}_{\star} \boldsymbol{y}\right)}
\end{aligned}
$$

Let $\boldsymbol{w}=\sqrt{N} \boldsymbol{y}=\sqrt{N}[\boldsymbol{x}-\boldsymbol{z}]$ so that $\boldsymbol{w} \in N^{-1 / 2} \mathbb{Z}^{d}$, to rewrite the previous sum as

$$
\left[1+o_{N}(1)\right] \frac{\mu}{2 \pi N} \sum_{\boldsymbol{w}} e^{-(1 / 2)\left(\boldsymbol{w} \cdot \mathbb{M}_{\star} \boldsymbol{w}\right)},
$$

where the sum is performed over $\boldsymbol{w}$ such that $|\boldsymbol{w} \cdot \boldsymbol{v}| \leq N^{1 / 2} \varepsilon_{N}$ and $\left|\boldsymbol{w} \cdot \boldsymbol{w}^{j}\right| \leq$ $2 \sqrt{\mu / \lambda_{j}} N^{1 / 2} \varepsilon_{N}, 2 \leq j \leq d$. Since, by assumption, $N^{1 / 2} \varepsilon_{N} \uparrow \infty$, this expression is equal to

$$
\left[1+o_{N}(1)\right] \frac{\mu}{2 \pi N} N^{d / 2} \int_{\mathbb{R}^{d}} e^{-(1 / 2)\left(\boldsymbol{w} \cdot \mathbb{M}_{\star} \boldsymbol{w}\right)} d \boldsymbol{w} .
$$

The previous integral is equal to $(2 \pi)^{d / 2}\left\{\operatorname{det} \mathbb{M}_{\star}\right\}^{-1 / 2}=(2 \pi)^{d / 2}\{-\operatorname{det} \mathbb{M}\}^{-1 / 2}$, which completes the proof of the assertion.

We conclude the proof of Proposition 3.1 extending the definition of $V_{N}$ to the entire set $\Xi_{N}$ and estimating its Dirichlet form. We denote by $\partial^{\text {in }} B_{N}$ the inner boundary of $B_{N}$, the set of points in $B_{N}$ which have a neighbor in $\Xi_{N} \backslash B_{N}$. Let $B_{N}^{*}, \partial_{ \pm}^{\text {in }} B_{N}$ be the $(d-1)$-dimensional sections of the boundary $\partial B_{N}, \partial^{\text {in }} B_{N}$ :

$$
\begin{aligned}
B_{N}^{*}= & \bigcup_{2 \leq j \leq d}\left\{\boldsymbol{x} \in \partial B_{N}:\left|(\boldsymbol{x}-\boldsymbol{z}) \cdot \boldsymbol{w}^{j}\right|>2 \sqrt{\mu / \lambda_{j}} \varepsilon_{N}\right\}, \\
& \partial_{ \pm}^{\text {in }} B_{N}=\left\{\boldsymbol{x} \in \partial^{\text {in }} B_{N}: \exists \boldsymbol{y} \in \partial_{ \pm} B_{N} \text { s.t. }\|\boldsymbol{y}-\boldsymbol{x}\|=N^{-1}\right\} .
\end{aligned}
$$


Assertion 3.C. For all $N$ sufficiently large,

$$
\inf _{\boldsymbol{x} \in B_{N}^{*}} F(\boldsymbol{x}) \geq F(\boldsymbol{z})+\mu \varepsilon_{N}^{2} .
$$

Proof. Indeed, by a Taylor expansion of $F$ around $\boldsymbol{z}$, for $\boldsymbol{x} \in B_{N}^{*}$,

$$
F(\boldsymbol{x})=F(\boldsymbol{z})+(1 / 2)(\boldsymbol{x}-\boldsymbol{z}) \cdot \mathbb{M}(\boldsymbol{x}-\boldsymbol{z})+O\left(\varepsilon_{N}^{3}\right) .
$$

The second term on the right hand side is equal to $(1 / 2) \sum_{1<j<d} \lambda_{j}\left[(\boldsymbol{x}-\boldsymbol{z}) \cdot \boldsymbol{w}^{j}\right]^{2}$. Since $\lambda_{1}=-\mu, \lambda_{j}>0$ for $2 \leq j \leq d$, and $\boldsymbol{x}$ belongs to $B_{N}^{*}$, for $N$ sufficiently large the previous expression is bounded below by

$$
F(\boldsymbol{z})+\frac{3 \mu}{2} \varepsilon_{N}^{2}+O\left(\varepsilon_{N}^{3}\right) \geq F(\boldsymbol{z})+\mu \varepsilon_{N}^{2},
$$

which proves the claim.

Let $\vartheta=\min \{\mu(\boldsymbol{z}): \boldsymbol{z} \in \mathfrak{S}(A)\}$. Denote by $\mathcal{U}$ the connected component of the set $\left\{\boldsymbol{x} \in \Xi: F(\boldsymbol{x})<F(\boldsymbol{z})+\vartheta \varepsilon_{N}^{2}\right\}$ which contains a set $W_{a}, a \in A$. The set $\mathcal{U}$ may be decomposed in disjoint sets. Recall from $(3.2)$ the definition of the sets $\mathcal{B}^{z}$, $\boldsymbol{z} \in \mathfrak{S}(A)$, and let $\mathcal{V}=\mathcal{U} \backslash \cup_{\boldsymbol{z} \in \mathfrak{S}(A)} \mathcal{B}^{z}$. Figure 3 represents the sets $\mathcal{U}$ and $\mathcal{B}^{z}$. By Assertion 3.C the set $\mathcal{V}$ is formed by several connected components separated by the sets $\mathcal{B}^{z}, z \in \mathfrak{S}(A)$. In Figure 3 , for example, the set $\mathcal{V}$ is composed of 4 connected components.

Let $\mathcal{U}_{N}=\mathcal{U} \cap \Xi_{N}, \mathfrak{B}_{N}^{z}=\mathcal{U}_{N} \cap \mathcal{B}^{z}, \boldsymbol{z} \in \mathfrak{S}(A), \mathcal{V}_{N}=\mathcal{U}_{N} \backslash \cup_{\boldsymbol{z} \in \mathfrak{S}(A)} \mathfrak{B}_{N}^{z}$ so that

$$
\mathcal{U}_{N}=\mathcal{V}_{N} \cup \bigcup_{\boldsymbol{z} \in \mathfrak{S}(A)} \mathfrak{B}_{N}^{z}
$$

Let $\mathcal{V}_{N}^{A}$ be the union of all connected components of $\mathcal{V}_{N}$ which contains a point in $W_{a}, a \in A$, and let $\mathcal{V}_{N}^{B}=\mathcal{V}_{N} \backslash \mathcal{V}_{N}^{A}$.

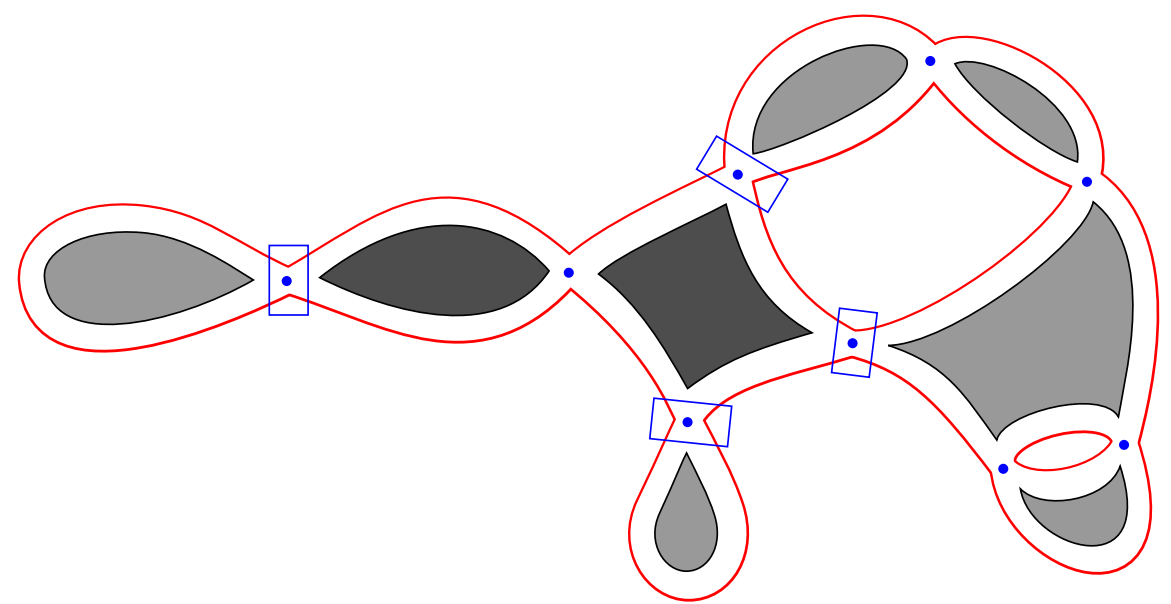

FiguRE 3 . In red the boundary of the set $\mathcal{U}$. In dark gray the wells $W_{b}^{\epsilon}$, $b \in A$. In blue the boxes $\mathcal{B}^{\boldsymbol{z}}, \boldsymbol{z} \in \mathfrak{S}(A)$.

For each $\boldsymbol{z} \in \mathfrak{S}(A)$, choose an orthonormal basis of (Hess $F)(\boldsymbol{z})$ in such a way that the eigenvector $\boldsymbol{v}(\boldsymbol{z})$ points to the direction of $\mathcal{E}_{N}(A)$. Define $V_{N}^{A}: \Xi_{N} \rightarrow[0,1]$ 
by

$$
V_{N}^{A}(\boldsymbol{x})=\left\{\begin{array}{ll}
0 & \boldsymbol{x} \in \mathcal{V}_{N}^{B}, \\
1 & \boldsymbol{x} \in \mathcal{V}_{N}^{A},
\end{array} \quad V_{N}^{A}(\boldsymbol{x})= \begin{cases}V_{N}^{\boldsymbol{z}}(\boldsymbol{x}) & \boldsymbol{x} \in \mathfrak{B}_{N}^{\boldsymbol{z}} \\
(1 / 2) & \text { otherwise }\end{cases}\right.
$$

where $V_{N}^{\boldsymbol{z}}$ is the function defined in 3.5 .

Assertion 3.D. Let $\varepsilon_{N}$ be a sequence such that $N \varepsilon_{N}^{3} \rightarrow 0, \exp \left\{-N \varepsilon_{N}^{2}\right\}$ converges to 0 faster than any polynomial. Then,

$$
\frac{Z_{N}}{(2 \pi N)^{d / 2}} 2 \pi N e^{N F(\boldsymbol{z})} D_{N}\left(V_{N}^{A}\right) \leq\left[1+o_{N}(1)\right] \sum_{\boldsymbol{z} \in \mathfrak{S}(A)} \frac{\mu(\boldsymbol{z})}{\sqrt{-\operatorname{det} \operatorname{Hess} F(\boldsymbol{z})}} .
$$

Proof. We estimate the Dirichlet form of $V_{N}^{A}$ inside the sets $\mathfrak{B}_{N}^{\boldsymbol{z}}, \boldsymbol{z} \in \mathfrak{S}(A)$, at the boundary of $\mathcal{U}_{N}$, and at the boundary of $B_{N}^{z}$ which is contained in $\mathcal{U}_{N}$.

Denote by $\partial \mathcal{U}_{N}$ the outer boundary of $\mathcal{U}_{N}$. The contribution to the Dirichlet form $D_{N}\left(V_{N}^{A}\right)$ of the edges in $\partial \mathcal{U}_{N}$ is less than or equal to

$$
\sum_{i=1}^{d} \sum_{\boldsymbol{x} \in \partial \mathcal{U}_{N}} \mu_{N}(\boldsymbol{x})\left[R_{N}\left(\boldsymbol{x}, \boldsymbol{x}+\boldsymbol{e}_{i}\right)+R_{N}\left(\boldsymbol{x}, \boldsymbol{x}-\boldsymbol{e}_{i}\right)\right] \leq \frac{C_{0}}{Z_{N}} e^{-N F(\boldsymbol{z})} \sum_{x \in \partial \mathcal{U}_{N}} e^{-\vartheta N \varepsilon_{N}^{2}},
$$

where $C_{0}$ denotes a finite constant which does not depend on $N$ and whose value may change from line to line. The sum on the right hand side is bounded by $C_{0} N^{d-1} e^{-\vartheta N \varepsilon_{N}^{2}}$, which vanishes as $N \uparrow \infty$ in view of our choice of $\varepsilon_{N}$.

Let $R_{N}^{ \pm}(\boldsymbol{z})=\partial_{ \pm}^{\text {in }} B_{N}^{\boldsymbol{z}} \cap \mathcal{U}_{N}, \boldsymbol{z} \in \mathfrak{S}(A)$. we estimate the contribution to the Dirichlet form $D_{N}\left(V_{N}^{A}\right)$ of the edges in $R_{N}^{-}(\boldsymbol{z})$, the one of $R_{N}^{+}(\boldsymbol{z})$ being analogous. By the definition of $V_{N}^{A}$ this contribution is bounded by

$$
\begin{gathered}
\sum_{i=1}^{d} \sum_{\boldsymbol{x} \in R_{N}^{-}(\boldsymbol{z})} \mu_{N}(\boldsymbol{x})\left[R_{N}\left(\boldsymbol{x}, \boldsymbol{x}+\boldsymbol{e}_{i}\right)+R_{N}\left(\boldsymbol{x}, \boldsymbol{x}-\boldsymbol{e}_{i}\right)\right] V_{N}^{\boldsymbol{z}}(\boldsymbol{x})^{2} \\
\leq \frac{C_{0}}{Z_{N}} e^{-N F(\boldsymbol{z})} \sum_{\boldsymbol{x} \in R_{N}^{-}(\boldsymbol{z})} e^{-(1 / 2) N\left(\boldsymbol{y} \cdot \mathbb{M}^{\boldsymbol{z}} \boldsymbol{y}\right)} V_{N}^{\boldsymbol{z}}(\boldsymbol{x})^{2}
\end{gathered}
$$

where $\boldsymbol{y}=\boldsymbol{x}-\boldsymbol{z}$. In the remainder of this paragraph we omit the dependence on $\boldsymbol{z}$ in the notation. Since $\boldsymbol{x}$ belongs to $B_{N}, \exp \{-(1 / 2) N(\boldsymbol{y} \cdot \mathbb{M} \boldsymbol{y})\}$ is less than or equal to $\exp \left\{(1 / 2) \mu N \varepsilon_{N}^{2}\right\} \exp \left\{-(1 / 2) N \sum_{2 \leq j \leq d} \lambda_{j}\left(\boldsymbol{y} \cdot \boldsymbol{w}^{j}\right)^{2}\right\}$. On the other hand, by a change of variables,

$$
V_{N}(\boldsymbol{x})^{2}=\left(\frac{1}{\sqrt{2 \pi}} \int_{-\infty}^{(N \mu)^{1 / 2}(\boldsymbol{y} \cdot \boldsymbol{v})} e^{-r^{2} / 2} d r\right)^{2}
$$

Since $\boldsymbol{x}$ belongs to $\partial_{-}^{\text {in }} B_{N}, \boldsymbol{y} \cdot \boldsymbol{v} \leq-\varepsilon_{N}+C_{0} N^{-1}$. The previous expression is therefore less than or equal to $\left(C_{0} / N \varepsilon_{N}^{2}\right) \exp \left\{-\mu N \varepsilon_{N}^{2}\right\}$ because $\int_{(-\infty, A]} \exp \left\{-(1 / 2) r^{2}\right\} d r$ $\leq|A|^{-1} \exp \left\{-(1 / 2) A^{2}\right\}$ for $A<0$. This proves that the sum appearing in 3.6 is less than or equal to $C_{0} N^{d-1} \exp \left\{-(1 / 2) \mu N \varepsilon_{N}^{2}\right\}$, which vanishes as $N \uparrow \infty$, in view of the definition of $\varepsilon_{N}$.

Since, for each $\boldsymbol{z} \in \mathfrak{S}(A)$, the set $\mathfrak{B}_{N}^{\boldsymbol{z}}$ is contained in $B_{N}^{\boldsymbol{z}}$, the contribution to the Dirichlet form of the bonds in the set $\mathfrak{B}_{N}^{\boldsymbol{z}}$ is less than or equal to $D_{N}\left(V_{N} ; B_{N}^{\boldsymbol{z}}\right)$. To conclude the proof it remains to recall Assertion 3.B. 


\section{LOWER BOUND FOR THE CAPACITIES}

We prove in this section the lower bound of Theorem 2.1. The proof is based on the arguments presented in [5, 6].

Proposition 4.1. For every proper subset $A$ of $\{1, \ldots, \ell\}$,

$\liminf _{N \rightarrow \infty} \frac{Z_{N}}{(2 \pi N)^{d / 2}} 2 \pi N e^{N F(\boldsymbol{z})} \operatorname{cap}_{N}\left(\mathcal{E}_{N}(A), \mathcal{E}_{N}\left(A^{c}\right)\right) \geq \sum_{\boldsymbol{z} \in \mathfrak{S}(A)} \frac{\mu(\boldsymbol{z})}{\sqrt{-\operatorname{det} \operatorname{Hess} F(\boldsymbol{z})}}$.

The idea of the proof is quite simple. It is based on Thomson's principle [16. Proposition 3.2.2] which expresses the inverse of the capacity as an infimum over divergence free, unitary flows. The construction of a unitary flow from $\varepsilon_{N}(A)$ to $\mathcal{E}_{N}\left(A^{c}\right)$ will be done in two steps. We first construct a unitary flow from $\mathcal{E}_{N}(A)$ to $\mathcal{E}_{N}\left(A^{c}\right)$ for each saddle point $\boldsymbol{z} \in \mathfrak{S}(A)$. Then, we define a unitary flow from $\mathcal{E}_{N}(A)$ to $\mathcal{E}_{N}\left(A^{c}\right)$ as a convex combination of the unitary flows defined in the first step.

Step 1: Flows associated to saddle points. The main difficulty of the proof of Proposition 4.1 consists in defining unitary flows associated to saddle points. Fix $\boldsymbol{z} \in \mathfrak{S}(A)$ and two wells $W_{a}, W_{b}$ such that $a \in A, b \in A^{c}, \boldsymbol{z} \in W_{a} \cap W_{b}$. Assume, without loss of generality, that all coordinates of the vector $\boldsymbol{v}$ are non-negative. Let $B_{N}$ be the subset defined by

$$
B_{N}=\left\{\boldsymbol{x} \in \Xi_{N}:|(\boldsymbol{x}-\boldsymbol{z}) \cdot \boldsymbol{v}| \leq \varepsilon_{N}, \max _{2 \leq j \leq d}\left|(\boldsymbol{x}-\boldsymbol{z}) \cdot \boldsymbol{w}^{j}\right| \leq \varepsilon_{N}\right\},
$$

where $\varepsilon_{N}$ is a sequence such that $N \varepsilon_{N}^{3} \rightarrow 0, \exp \left\{-N \varepsilon_{N}^{2}\right\}$ converges to 0 faster than any polynomial. Note that the definition of the set $B_{N}$ changed with respect to the one of the previous section.

Keep in mind that we assumed $\boldsymbol{v}$ to be a vector with non-negative coordinates. Denote by $N(\boldsymbol{v})$ the set of positive coordinates of $\boldsymbol{v}, N(\boldsymbol{v})=\left\{j: \boldsymbol{v}_{j}>0\right\}$. Let $Q_{N}^{o}$ be the cone $Q_{N}^{o}=\left\{\boldsymbol{x} \in N^{-1} \mathbb{Z}^{d}: \boldsymbol{x}_{j} \geq 0, j \in N(\boldsymbol{v})\right.$ and $\left.\boldsymbol{x}_{j}=0, j \notin N(\boldsymbol{v})\right\}$, and let $Q_{N}^{\boldsymbol{x}}, \boldsymbol{x} \in N^{-1} \mathbb{Z}^{d}$, be the cone $Q_{N}^{o}$ translated by $\boldsymbol{x}, Q_{N}^{\boldsymbol{x}}=\left\{\boldsymbol{x}+\boldsymbol{x}^{\prime}: \boldsymbol{x}^{\prime} \in Q_{N}^{o}\right\}$.

Denote by $\partial_{-}^{\text {in }} B_{N}$ the inner boundary of $B_{N}$, defined as $\partial_{-}^{\text {in }} B_{N}=\left\{\boldsymbol{x} \in B_{N}\right.$ : $\exists j$ s.t. $\left.\left[\boldsymbol{x}-\boldsymbol{z}-\boldsymbol{e}_{j}\right] \cdot \boldsymbol{v}<-\varepsilon_{N}\right\}$. Denote by $Q_{N}^{+}$the set of all cones with root in $\partial_{-}^{\text {in }} B_{N}, Q_{N}^{+}=\cup_{x \in \partial_{-}^{\text {in }} B_{N}} Q_{N}^{x}$, and let

$$
Q_{N}=\left\{\boldsymbol{x} \in Q_{N}^{+}:(\boldsymbol{x}-\boldsymbol{z}) \cdot \boldsymbol{v} \leq \varepsilon_{N}\right\} .
$$

Note that $B_{N} \subset Q_{N}$. Figure 4 represents the sets $B_{N}, Q_{N}$.

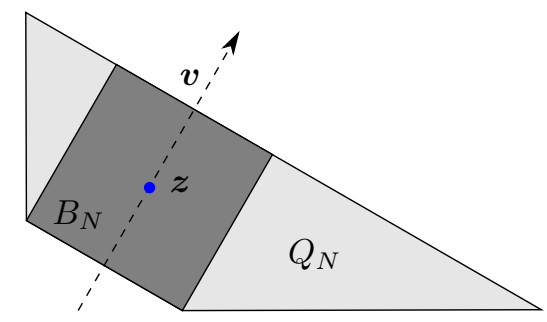

Figure 4. The vector $\boldsymbol{v}$, the set $B_{N}$ in dark gray, and the set $Q_{N}$ in light gray. 
There exists a finite constant $C_{0}$, independent of $N$, such that for all $N \geq 1$,

$$
\max _{2 \leq k \leq d} \max _{\boldsymbol{x} \in Q_{N}}\left|(\boldsymbol{x}-\boldsymbol{z}) \cdot \boldsymbol{w}^{k}\right| \leq C_{0} \varepsilon_{N} .
$$

Indeed, if $\boldsymbol{x}$ belongs to $Q_{N}, \boldsymbol{x}=\boldsymbol{x}^{\prime}+\boldsymbol{x}^{\prime \prime}$, where $\boldsymbol{x}^{\prime} \in \partial_{-}^{\text {in }} B_{N}$ and $\boldsymbol{x}^{\prime \prime} \in Q_{N}^{o}$. On the one hand, $\boldsymbol{x}^{\prime} \in B_{N}$ so that $\left|\left(\boldsymbol{x}^{\prime}-\boldsymbol{z}\right) \cdot \boldsymbol{w}^{k}\right| \leq \varepsilon_{N}$ for all $k$ and $N$. On the other hand, $\boldsymbol{x}^{\prime \prime} \cdot \boldsymbol{v}=[\boldsymbol{x}-\boldsymbol{z}] \cdot \boldsymbol{v}-\left[\boldsymbol{x}^{\prime}-\boldsymbol{z}\right] \cdot \boldsymbol{v}$. The first term is bounded by $\varepsilon_{N}$ because $\boldsymbol{x}$ belongs to $Q_{N}$. As $\boldsymbol{x}^{\prime} \in B_{N}$, the second term is absolutely bounded by $\varepsilon_{N}$. This proves that $\boldsymbol{x}_{j}^{\prime \prime} \leq C_{0} \varepsilon_{N}$ for all $j \in N(\boldsymbol{v})$. The inequality holds trivially for $j \notin N(\boldsymbol{v})$ from what we conclude that there exists $C_{0}$ such that $\boldsymbol{x}_{j}^{\prime \prime} \leq C_{0} \varepsilon_{N}$ for all $j$ and $N$. Assertion (4.1) follows from this bound and from the bounds obtained on $\boldsymbol{x}^{\prime}$.

Denote by $\partial B_{N}$ the external boundary of the set $B_{N}$, the set of sites which do not belong to $B_{N}$ and which have a neighbor in $B_{N}: \partial B_{N}=\left\{\boldsymbol{x} \notin B_{N}\right.$ : $\exists j$ s.t. $\boldsymbol{x}+\boldsymbol{e}_{j}$ or $\left.\boldsymbol{x}-\boldsymbol{e}_{j} \in B_{N}\right\}$. Two pieces of the external boundary of $B_{N}$ play an important role in the proof of the lower bound for the capacity. Denote by $\partial_{ \pm} B_{N}$ the sets

$\partial_{-} B_{N}=\left\{\boldsymbol{x} \in \partial B_{N}:(\boldsymbol{x}-\boldsymbol{z}) \cdot \boldsymbol{v}<-\varepsilon_{N}\right\}, \quad \partial_{+} B_{N}=\left\{\boldsymbol{x} \in \partial B_{N}:(\boldsymbol{x}-\boldsymbol{z}) \cdot \boldsymbol{v}>\varepsilon_{N}\right\}$.

Denote by $\partial_{+} Q_{N}$ the outer boundary of $Q_{N}$ defined by $\partial_{+} Q_{N}=\left\{\boldsymbol{x} \in \Xi_{N}\right.$ : $[\boldsymbol{x}-\boldsymbol{z}] \cdot \boldsymbol{v}>\varepsilon_{N}$ and $\exists j$ s.t. $\left.\boldsymbol{x}-\boldsymbol{e}_{j} \in Q_{N}\right\}$. We shall construct a divergence free, unitary flow from $\varepsilon_{N}^{a}$ to $\partial_{-} B_{N}$, one from $\partial_{-} B_{N}$ to $\partial_{+} Q_{N}$ and a third one from $\partial_{+} Q_{N}$ to $\varepsilon_{N}^{b}$. The more demanding one is the flow from $\partial_{-} B_{N}$ to $\partial_{+} Q_{N}$.

1.A. Sketch of the proof. To explain the idea of the proof of this part, we first consider the case where the eigenvector $\boldsymbol{v}$ associated to the negative eigenvalue of $(\operatorname{Hess} F)(\boldsymbol{z})$ is $\mathfrak{e}_{1}$, the first vector of the canonical basis. In this case the cone $Q_{N}^{o}$ introduced in the previous section is just a "straight line": $Q_{N}^{o}=\{(k / N, 0, \ldots, 0)$ : $k \geq 0\}$ and $\partial_{+} Q_{N}$ coincides with $\partial_{+} B_{N}$.

We know that the optimal unitary flow from $\partial_{-} B_{N}$ to $\partial_{+} B_{N}$ is given by $\widehat{\Phi}(\boldsymbol{x}, \boldsymbol{y})=$ $c(\boldsymbol{x}, \boldsymbol{y})[\widehat{V}(\boldsymbol{x})-\widehat{V}(\boldsymbol{y})] / \operatorname{cap}\left(\partial_{-} B_{N}, \partial_{+} B_{N}\right)$, where $c(\boldsymbol{x}, \boldsymbol{y})=\mu_{N}(\boldsymbol{x}) R_{N}(\boldsymbol{x}, \boldsymbol{y})$ is the conductance between the vertices $\boldsymbol{x}$ and $\boldsymbol{y}$ and $\widehat{V}$ is the equilibrium potential between $\partial_{-} B_{N}$ and $\partial_{+} B_{N}$. We introduced in (3.5) an approximation $V$ of the equilibrium potential $\widehat{V}$. A calculation shows that the flow $\Phi(\boldsymbol{x}, \boldsymbol{y})=c(\boldsymbol{x}, \boldsymbol{y})[V(\boldsymbol{x})-V(\boldsymbol{y})]$ is almost constant along the $\boldsymbol{v}$ direction. Hence, in the case where $\boldsymbol{v}=\mathfrak{e}_{1}$, a natural candidate is a flow constant along the $\mathfrak{e}_{1}$ direction. Denote a point $\boldsymbol{x} \in \Xi_{N}$ as $(\hat{\boldsymbol{x}}, \check{\boldsymbol{x}})$ where $\hat{\boldsymbol{x}} \in N^{-1} \mathbb{Z}$ and $\check{\boldsymbol{x}} \in N^{-1} \mathbb{Z}^{d-1}$, and let $\check{B}_{N}=\left\{\check{\boldsymbol{x}} \in N^{-1} \mathbb{Z}^{d-1}: \exists x \in\right.$ $N^{-1} \mathbb{Z}$ s.t. $\left.(x, \check{\boldsymbol{x}}) \in B_{N}\right\}$

$$
\Phi(\boldsymbol{x}, \boldsymbol{y})= \begin{cases}\Phi(\check{\boldsymbol{x}}) & \text { if } \boldsymbol{y}=\boldsymbol{x}+\boldsymbol{e}_{1}, \boldsymbol{x} \in B_{N} \cup \partial_{-} B_{N} \\ 0 & \text { otherwise }\end{cases}
$$

where $\Phi: \check{B}_{N} \rightarrow \mathbb{R}_{+}$is such that $\sum_{\boldsymbol{x} \in \check{B}_{N}} \Phi(\check{\boldsymbol{x}})=1$.

By Thomson's principle, the inverse of the capacity is bounded above by the energy dissipated by the flow $\Phi$ :

$$
\frac{1}{\operatorname{cap}_{N}\left(\partial_{-} B_{N}, \partial_{+} B_{N}\right)} \leq\|\Phi\|^{2}:=\sum_{\boldsymbol{x} \in B_{N} \cup \partial_{-} B_{N}} \frac{1}{c\left(\boldsymbol{x}, \boldsymbol{x}+\boldsymbol{e}_{1}\right)} \Phi\left(\boldsymbol{x}, \boldsymbol{x}+\boldsymbol{e}_{1}\right)^{2}
$$


By definition of the flow and by a second order Taylor expansion, the previous sum is equal to

$$
\left[1+o_{N}(1)\right] Z_{N} e^{N F(\boldsymbol{z})} \sum_{\boldsymbol{x} \in B_{N} \cup \partial_{-} B_{N}} e^{(N / 2)(\boldsymbol{y} \cdot \mathbb{M} \boldsymbol{y})} \Phi(\check{\boldsymbol{x}})^{2},
$$

provided $N \varepsilon_{N}^{3} \rightarrow 0$. In this equation, $\boldsymbol{y}=\boldsymbol{x}-\boldsymbol{z}$. Recall from (3.1) the definition of the matrices $\mathbb{V}, \mathbb{D}$. Let $\check{\mathbb{D}}$ be the diagonal matrix in which the entry $\lambda_{1}=-\mu$ has been replaced by 0 , and let $\check{\mathbb{M}}$ be the symmetric matrix $\check{\mathbb{M}}=\mathbb{V} \check{D} \mathbb{V}^{*}$. In particular, for any vector $\boldsymbol{y}, \boldsymbol{y} \cdot \check{\mathbb{M}} \boldsymbol{y}=\sum_{2 \leq k \leq d} \lambda_{k}\left(\boldsymbol{y} \cdot \boldsymbol{w}^{k}\right)^{2}$, and $\boldsymbol{y} \cdot \mathbb{M} \boldsymbol{y}=\boldsymbol{y} \cdot \check{\mathbb{M}} \boldsymbol{y}-\mu \hat{\boldsymbol{y}}^{2}$. With this notation, and since $\boldsymbol{y} \cdot \check{\mathbb{M}} \boldsymbol{y}$ depends on $\boldsymbol{y}$ only as a function of $\check{\boldsymbol{y}}$, we may rewrite the previous sum as

$$
\left[1+o_{N}(1)\right] Z_{N} e^{N F(\boldsymbol{z})} \sum_{\check{\boldsymbol{x}} \in \check{B}_{N}} e^{(N / 2)(\boldsymbol{y} \cdot \check{\mathbb{M}} \boldsymbol{y})} \Phi(\check{\boldsymbol{x}})^{2} \sum_{k} e^{-\mu(N / 2) k^{2}},
$$

where the second sum is performed over all $k \in N^{-1} \mathbb{Z}$ such that $-\varepsilon_{N}-N^{-1} \leq k \leq$ $\varepsilon_{N}$. The optimal choice of $\Phi$ satisfying $\sum_{\boldsymbol{x} \in \check{B}_{N}} \Phi(\check{\boldsymbol{x}})=1$ is

$$
\Phi(\check{\boldsymbol{x}})=e^{-(N / 2)(\boldsymbol{y} \cdot \check{\mathbb{M}} \boldsymbol{y})} / \sum_{\boldsymbol{x} \in \check{B}_{N}} e^{-(N / 2)(\boldsymbol{y} \cdot \check{\mathbb{M}} \boldsymbol{y})} .
$$

With this choice the previous sum becomes

$$
\left[1+o_{N}(1)\right] Z_{N} e^{N F(\boldsymbol{z})} \frac{\sum_{k} e^{-\mu(N / 2) k^{2}}}{\sum_{\check{\boldsymbol{x}} \in \check{B}_{N}} e^{-(N / 2)(\boldsymbol{y} \cdot \check{\mathbb{M}} \boldsymbol{y})}} .
$$

At this point we may repeat the arguments presented at the end of the proof of Assertion $3 . \mathrm{B}$ to conclude that the previous expression is equal to

$\left[1+o_{N}(1)\right] Z_{N} e^{N F(\boldsymbol{z})} \frac{(2 \pi N) \sqrt{-\operatorname{det} \mathbb{M} / \mu}}{(2 \pi N)^{d / 2} \sqrt{\mu}}=\left[1+o_{N}(1)\right] Z_{N} e^{N F(\boldsymbol{z})} \frac{(2 \pi N) \sqrt{-\operatorname{det} \mathbb{M}}}{(2 \pi N)^{d / 2} \mu}$,

In conclusion, we constructed a divergence free, unitary flow $\Phi$ from $\partial_{-} B_{N}$ to $\partial_{+} B_{N}$ whose dissipated energy, $\|\Phi\|^{2}$, defined in (4.2) satisfies

$$
\lim _{N \rightarrow \infty} \frac{(2 \pi N)^{d / 2}}{Z_{N}} \frac{1}{2 \pi N} e^{-N F(\boldsymbol{z})}\|\Phi\|^{2}=\frac{\sqrt{-\operatorname{det}[(\operatorname{Hess} F)(\boldsymbol{z})]}}{\mu} .
$$

1.B. A unitary flow from $\partial_{-} B_{N}$ to $\partial_{+} Q_{N}$. We turn now to the general case. We learned from the previous example that the optimal flow is $\Phi(\boldsymbol{x}, \boldsymbol{y})=$ $M_{N}^{-1} c(\boldsymbol{x}, \boldsymbol{y})[V(\boldsymbol{x})-V(\boldsymbol{y})]$, where $V$ is the function introduced in 3.5 and $M_{N}$ a constant which turns the flow unitary. We thus propose the flow

$$
\Phi\left(\boldsymbol{x}, \boldsymbol{x}+\boldsymbol{e}_{j}\right)=\frac{\sqrt{-\operatorname{det} \mathbb{M} / \mu}}{(2 \pi N)^{(d-1) / 2}} \boldsymbol{v}_{j} e^{-(N / 2) \boldsymbol{y} \cdot \check{\mathbb{M}} \boldsymbol{y}} .
$$

We claim that $\Phi$ is an essentially unitary flow:

$$
\sum_{j=1}^{d} \sum_{\boldsymbol{x} \in \partial_{j,-} B_{N}} \Phi\left(\boldsymbol{x}, \boldsymbol{x}+\boldsymbol{e}_{j}\right)=\left[1+o_{N}(1)\right]
$$

where $\partial_{j,-} B_{N}$ represents the set of points $\boldsymbol{x} \in \partial_{-} B_{N}$ such that $\boldsymbol{x}+\boldsymbol{e}_{j} \in B_{N}$. We have to show that

$$
\sum_{j=1}^{d} \boldsymbol{v}_{j} \sum_{\boldsymbol{x} \in \partial_{j,-} B_{N}} e^{-(N / 2) \boldsymbol{y} \cdot \check{\mathbb{M}} \boldsymbol{y}}=\left[1+o_{N}(1)\right] \frac{(2 \pi N)^{(d-1) / 2} \sqrt{\mu}}{\sqrt{-\operatorname{det} \mathbb{M}}} .
$$


Fix $1 \leq j \leq d$, and let $V=\left\{\boldsymbol{x} \in \mathbb{R}^{d}:[\boldsymbol{x}-\boldsymbol{z}] \cdot \boldsymbol{v}=-\varepsilon_{N}\right\}$. Denote by $\delta(\boldsymbol{x})$, $\boldsymbol{x} \in \partial_{j,-} B_{N}$, the amount needed to translate $\boldsymbol{x}$ in the $\boldsymbol{e}_{j}$-direction for $\boldsymbol{x}$ to belong to $V: \boldsymbol{x}+\delta(\boldsymbol{x}) \boldsymbol{e}_{j} \in V$. Observe that $\delta(\boldsymbol{x}) \in(0,1]$. Let $T(\boldsymbol{x})=\boldsymbol{x}+\delta(\boldsymbol{x}) \boldsymbol{e}_{j}$, $\boldsymbol{x} \in \partial_{j,-} B_{N}$. Since $\delta(\boldsymbol{x})$ is absolutely bounded by 1 ,

$\sum_{\boldsymbol{x} \in \partial_{j,-} B_{N}} e^{-(N / 2) \boldsymbol{y} \cdot \check{\mathbb{M}} \boldsymbol{y}}=\left[1+o_{N}(1)\right] \sum_{\boldsymbol{x} \in \partial_{j,-} B_{N}} \exp \left\{-(N / 2) \sum_{k=2}^{d} \lambda_{k}\left\{[T(\boldsymbol{x})-\boldsymbol{z}] \cdot \boldsymbol{w}^{k}\right\}^{2}\right\}$

Replacing $\boldsymbol{x}$ by $\sqrt{N} \boldsymbol{x}$, and approximating the sum appearing on the right hand side by a Riemann integral, the previous term becomes

$$
\begin{gathered}
{\left[1+o_{N}(1)\right] \boldsymbol{v}_{j} N^{(d-1) / 2} \prod_{k=2}^{d} \int_{-\sqrt{N} \varepsilon_{N}}^{\sqrt{N} \varepsilon_{N}} e^{-(1 / 2) \lambda_{k} r^{2}} d r} \\
=\left[1+o_{N}(1)\right] \boldsymbol{v}_{j}(2 \pi N)^{(d-1) / 2} \frac{\sqrt{\mu}}{\sqrt{-\operatorname{det} \mathbb{M}}},
\end{gathered}
$$

where $\boldsymbol{v}_{j}$ appeared to take into account the tilt of the hypersurface $V$. Multiplying the last term by $\boldsymbol{v}_{j}$ and summing over $j$ we get 4.5 because $\|\boldsymbol{v}\|=1$. This proves that the flow $\Phi$ is essentially unitary, as stated in (4.4).

1.C. Turning the flow divergence free. In this subsection, we add a correction $R$ to the flow $\Phi$ to turn it divergence free. We start with an estimate on the divergence of the flow $\Phi$. Denote by $(\operatorname{div} \Phi)(\boldsymbol{x})$ the divergence of the flow $\Phi$ at $\boldsymbol{x}$ :

$$
(\operatorname{div} \Phi)(\boldsymbol{x})=\sum_{j=1}^{d}\left\{\Phi\left(\boldsymbol{x}, \boldsymbol{x}+\boldsymbol{e}_{j}\right)-\Phi\left(\boldsymbol{x}-\boldsymbol{e}_{j}, \boldsymbol{x}\right)\right\} .
$$

We claim that there exists a finite constant $C_{0}$, independent of $N$, such that

$$
\max _{i \in N(\boldsymbol{v})} \max _{\boldsymbol{x} \in Q_{N}}\left|\frac{(\operatorname{div} \Phi)(\boldsymbol{x})}{\Phi\left(\boldsymbol{x}, \boldsymbol{x}+\boldsymbol{e}_{i}\right)}\right| \leq C_{0} \varepsilon_{N}^{2} .
$$

Fix $i \in N(\boldsymbol{v}), \boldsymbol{x} \in Q_{N}$, and recall the definition of the flow $\Phi$. By (4.1), by definition of the matrix $\check{\mathbb{M}}$ and by a second order Taylor expansion, for each $1 \leq i \leq d$,

$$
\begin{aligned}
\sum_{j=1}^{d} \frac{\Phi\left(\boldsymbol{x}, \boldsymbol{x}+\boldsymbol{e}_{j}\right)-\Phi\left(\boldsymbol{x}-\boldsymbol{e}_{j}, \boldsymbol{x}\right)}{\Phi\left(\boldsymbol{x}, \boldsymbol{x}+\boldsymbol{e}_{i}\right)} & =\sum_{j=1}^{d} \frac{\boldsymbol{v}_{j}}{\boldsymbol{v}_{i}} \sum_{k=2}^{d} \lambda_{k}\left(\mathfrak{e}_{j} \cdot \boldsymbol{w}^{k}\right)\left([\boldsymbol{x}-\boldsymbol{z}] \cdot \boldsymbol{w}^{k}\right)+O\left(\varepsilon_{N}^{2}\right) \\
& =\frac{1}{\boldsymbol{v}_{i}} \sum_{k=2}^{d} \lambda_{k}\left(\boldsymbol{v} \cdot \boldsymbol{w}^{k}\right)\left([\boldsymbol{x}-\boldsymbol{z}] \cdot \boldsymbol{w}^{k}\right)+O\left(\varepsilon_{N}^{2}\right) .
\end{aligned}
$$

The first term on the right hand side vanishes because $\boldsymbol{v}$ is orthogonal to $\boldsymbol{w}^{k}$, which proves 4.6.

We now define a correction $R$ to the flow $\Phi$ to turn it divergence free. Let $G_{0}=\partial_{-} B_{N}, G$ for generation. Define recursively the sets $G_{k}, k \geq 1$, by

$$
G_{k+1}=\left\{\boldsymbol{x} \in Q_{N}: \boldsymbol{x}-\boldsymbol{e}_{j} \in \bigcup_{\ell=0}^{k} G_{\ell} \cup Q_{N}^{c} \text { for all } j \in N(\boldsymbol{v})\right\}, \quad k \geq 0 .
$$

The first three generations are illustrated in Figure 5 . Denote by $K_{N}$ the smallest integer $k$ such that $Q_{N} \subset \cup_{1 \leq \ell \leq k} G_{\ell}$. Clearly, $K_{N} \leq C_{0} \varepsilon_{N}^{-1}$ for some finite constant $C_{0}$. 


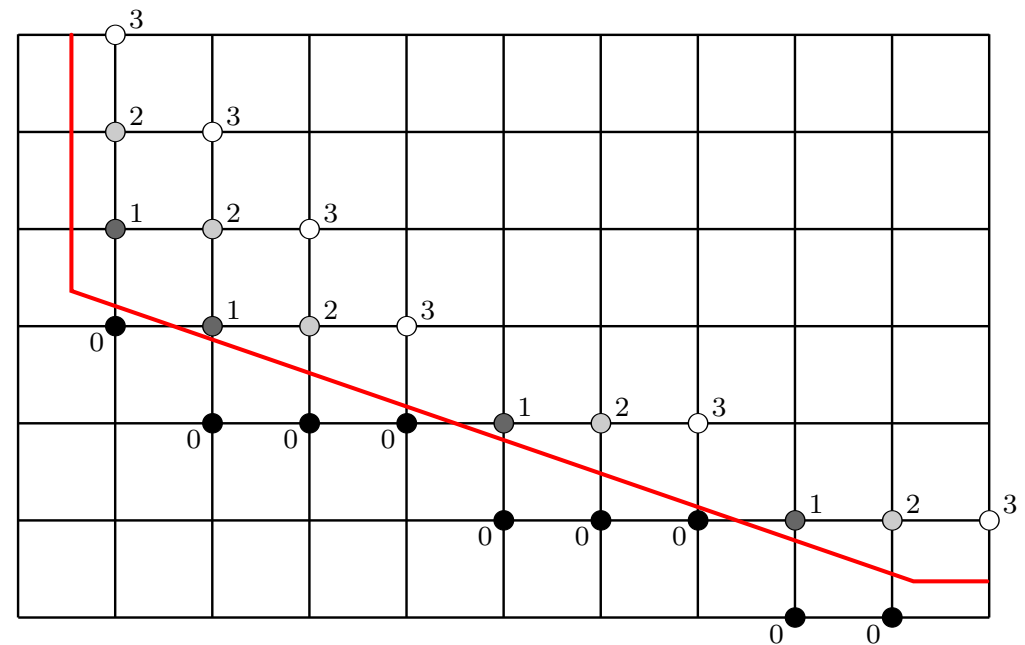

Figure 5. The first three generations. The red line represents the boundary of the set $Q_{N}$, and the numbers the generation of each point.

The flow $R$ is also defined recursively. For all $\boldsymbol{x} \in G_{1}$, define $R\left(\boldsymbol{x}-\boldsymbol{e}_{j}, \boldsymbol{x}\right)=0$, $1 \leq j \leq d$, and let

$$
R\left(\boldsymbol{x}, \boldsymbol{x}+\boldsymbol{e}_{j}\right)=p_{j}\left\{\sum_{i=1}^{d} R\left(\boldsymbol{x}-\boldsymbol{e}_{i}, \boldsymbol{x}\right)-(\operatorname{div} \Phi)(\boldsymbol{x})\right\},
$$

where $p_{j}=\boldsymbol{v}_{j} / \sum_{1 \leq i \leq d} \boldsymbol{v}_{i}$. Note that $R\left(\boldsymbol{x}, \boldsymbol{x}+\boldsymbol{e}_{j}\right)=0$ if $j \notin N(\boldsymbol{v})$ and that we may restrict the sum over $i$ to the set $N(\boldsymbol{v})$. On the other hand, by construction, $(\operatorname{div} R)(\boldsymbol{x})=-(\operatorname{div} \Phi)(\boldsymbol{x})$ for all $\boldsymbol{x} \in \cup_{1 \leq \ell \leq K_{N}} G_{\ell}$.

There exists a finite constant $C_{0}$ such that

$$
\max _{i \in N(\boldsymbol{v})} \max _{\boldsymbol{x} \in G_{k}}\left|\frac{R\left(\boldsymbol{x}, \boldsymbol{x}+\boldsymbol{e}_{i}\right)}{\Phi\left(\boldsymbol{x}, \boldsymbol{x}+\boldsymbol{e}_{i}\right)}\right| \leq C_{0} k \varepsilon_{N}^{2}
$$

for all $1 \leq k \leq K_{N}$. This assertion is proved by induction. Since $R\left(\boldsymbol{x}-\boldsymbol{e}_{j}, \boldsymbol{x}\right)=0$ for $\boldsymbol{x} \in G_{1}$, by (4.6), $\max _{i \in N(\boldsymbol{v})} \max _{\boldsymbol{x} \in G_{1}}\left|R\left(\boldsymbol{x}, \boldsymbol{x}+\boldsymbol{e}_{i}\right) / \Phi\left(\boldsymbol{x}, \boldsymbol{x}+\boldsymbol{e}_{i}\right)\right| \leq C_{1} \varepsilon_{N}^{2}$, where $C_{1}$ is the constant $C_{0}$ appearing on the right hand side of (4.6).

Suppose that $\max _{i \in N(\boldsymbol{v})} \max _{\boldsymbol{x} \in G_{j}}\left|R\left(\boldsymbol{x}, \boldsymbol{x}+\boldsymbol{e}_{i}\right) / \Phi\left(\boldsymbol{x}, \boldsymbol{x}+\boldsymbol{e}_{i}\right)\right| \leq C_{j} \varepsilon_{N}^{2}$ for all $1 \leq j \leq k$, where $C_{j}$ is an increasing sequence. Fix $i \in N(\boldsymbol{v})$ and $\boldsymbol{x} \in G_{k+1}$. By definition of $R$, by (4.6), and by the induction hypothesis,

$$
\left|\frac{R\left(\boldsymbol{x}, \boldsymbol{x}+\boldsymbol{e}_{i}\right)}{\Phi\left(\boldsymbol{x}, \boldsymbol{x}+\boldsymbol{e}_{i}\right)}\right| \leq C_{k} \varepsilon_{N}^{2} p_{i} \sum_{j \in N(\boldsymbol{v})} \frac{\Phi\left(\boldsymbol{x}-\boldsymbol{e}_{j}, \boldsymbol{x}\right)}{\Phi\left(\boldsymbol{x}, \boldsymbol{x}+\boldsymbol{e}_{i}\right)}+C_{0} \varepsilon_{N}^{2} .
$$

The computations performed to prove (4.6) yield that the first term on the right hand side is bounded by

$$
\begin{gathered}
C_{k} \varepsilon_{N}^{2} p_{i} \sum_{j \in N(\boldsymbol{v})} \frac{\boldsymbol{v}_{j}}{\boldsymbol{v}_{i}}\left\{1+\sum_{m=2}^{d} \lambda_{m}\left(\mathfrak{e}_{j} \cdot \boldsymbol{w}^{m}\right)\left(\boldsymbol{y} \cdot \boldsymbol{w}^{m}\right)+C_{0} \varepsilon_{N}^{2}\right\} \\
=C_{k} \varepsilon_{N}^{2}\left\{1+C_{0} \varepsilon_{N}^{2}\right\} \leq C_{k} \varepsilon_{N}^{2} e^{C_{0} \varepsilon_{N}^{2}} .
\end{gathered}
$$


The identity has been derived using the definition of $p_{j}$, the orthogonality of $\boldsymbol{v}$ and $\boldsymbol{w}^{k}$, and summing first over $j$. We have thus obtained the recursive relation $C_{k+1} \leq\left[C_{0}+C_{k} e^{C_{0} \varepsilon_{N}^{2}}\right]$ from which it follows that $C_{k} \leq C_{0} k e^{C_{0} k \varepsilon_{N}^{2}}$. Since $k \leq$ $K_{N} \leq C_{0} \varepsilon_{N}^{-1}, 4.8$ holds.

1.D. A divergence free unitary flow. We construct in this subsection a divergence-free, unitary flow from $\partial_{-} B_{N}$ to $\partial_{+} Q_{N}$ whose energy dissipated is given by the right hand side of 4.9 . .

Let $\Psi$ be the flow from $\partial_{-} B_{N}$ to $\partial_{+} Q_{N}$ defined by $\Psi=\Phi+R$, where $\Phi$ is introduced in (4.3) and $R$ in (4.7). By (4.4) and by construction of $R, \Psi$ is a unitary flow. Since $(\operatorname{div} R)(\boldsymbol{x})=-(\operatorname{div} \Phi)(\boldsymbol{x})$ for all $\boldsymbol{x} \in \cup_{0 \leq \ell \leq K_{N}} G_{\ell}, \Psi$ is divergence-free. It remains to show that the energy dissipated by $\Psi$ satisfies

$$
\begin{aligned}
& \sum_{j=1}^{d} \sum_{\boldsymbol{x}} \frac{1}{c\left(\boldsymbol{x}, \boldsymbol{x}+\boldsymbol{e}_{j}\right)} \Psi\left(\boldsymbol{x}, \boldsymbol{x}+\boldsymbol{e}_{j}\right)^{2} \\
& \quad=\left[1+o_{N}(1)\right] \frac{Z_{N}}{(2 \pi N)^{d / 2}} 2 \pi N e^{N F(\boldsymbol{z})} \frac{\sqrt{-\operatorname{det}[(\operatorname{Hess} F)(\boldsymbol{z})]}}{\mu} .
\end{aligned}
$$

A second order expansion of $F(\boldsymbol{x})$ at $\boldsymbol{z}$ taking advantage of (4.1) and of the fact that $N \varepsilon_{N}^{3} \rightarrow 0$ permits to write the left hand side of the previous equation as

$$
\left[1+o_{N}(1)\right] Z_{N} e^{N F(\boldsymbol{z})} \sum_{j=1}^{d} \sum_{\boldsymbol{x}} e^{(N / 2)(\boldsymbol{y} \cdot \mathbb{M} \cdot \boldsymbol{y})} \Psi\left(\boldsymbol{x}, \boldsymbol{x}+\boldsymbol{e}_{j}\right)^{2},
$$

where, as before, $\boldsymbol{y}=\boldsymbol{x}-\boldsymbol{z}$. We may bound $\Psi\left(\boldsymbol{x}, \boldsymbol{x}+\boldsymbol{e}_{j}\right)^{2}$ by $\left(1+\varepsilon_{N}\right) \Phi\left(\boldsymbol{x}, \boldsymbol{x}+\boldsymbol{e}_{j}\right)^{2}+$ $\left(1+\varepsilon_{N}^{-1}\right) R\left(\boldsymbol{x}, \boldsymbol{x}+\boldsymbol{e}_{j}\right)^{2}$, and apply (4.8) together with the fact that $k \leq K_{N} \leq C_{0} \varepsilon_{N}^{-1}$ to estimate the previous sum by $\left[1+O\left(\varepsilon_{N}\right)\right] \Phi\left(\boldsymbol{x}, \boldsymbol{x}+\boldsymbol{e}_{j}\right)^{2}$. The previous displayed equation is therefore equal to the same sum with $\Psi$ replaced by $\Phi$. Replacing $\Phi\left(\boldsymbol{x}, \boldsymbol{x}+\boldsymbol{e}_{j}\right)$ by its value 4.3 the previous sum becomes

$$
\left[1+o_{N}(1)\right] Z_{N} e^{N F(\boldsymbol{z})} \frac{1}{(2 \pi N)^{d-1}} \frac{\operatorname{det} \mathbb{M}}{-\mu} \sum_{j=1}^{d} \boldsymbol{v}_{j}^{2} \sum_{\boldsymbol{x}} e^{-(N / 2)\left(\boldsymbol{y} \cdot \mathbb{M}_{\star} \cdot \boldsymbol{y}\right)},
$$

where $\mathbb{M}_{\star}$ is the matrix introduced in (3.1). At this point it remains to recall that $\boldsymbol{v}$ is a normal vector and to repeat the calculations performed in the proof of the upper bound of the capacity to retrieve 4.9 ).

1.E. A unitary flow from $\varepsilon_{N}^{a}$ to $\partial_{-} B_{N}$. We extend in this section the flow $\Psi$ from $\partial_{-} B_{N}$ to $\varepsilon_{N}^{a}$. The same arguments permit to extend the flow $\Psi$ from $\partial_{+} Q_{N}$ to $\varepsilon_{N}^{b}$. The idea is quite simple. For each bond $\left(\boldsymbol{x}, \boldsymbol{x}+\boldsymbol{e}_{j}\right), \boldsymbol{x} \in \partial_{-} B_{N}, \boldsymbol{x}+\boldsymbol{e}_{j} \in B_{N}$, we construct a path of nearest neighbor sites $\left(\boldsymbol{x}=\boldsymbol{x}^{0}, \boldsymbol{x}^{1}, \ldots, \boldsymbol{x}^{n}\right), \boldsymbol{x}^{n} \in \mathcal{E}_{N}^{a}$, from $\boldsymbol{x}$ to $\mathcal{E}_{N}^{a}$, and we define the flow $\Psi_{\boldsymbol{x}, \boldsymbol{e}_{j}}$ from $\boldsymbol{x}$ to $\mathcal{E}_{N}^{a}$ by $\Psi_{\boldsymbol{x}, \boldsymbol{e}_{j}}\left(\boldsymbol{x}^{k}, \boldsymbol{x}^{k+1}\right)=-\Psi\left(\boldsymbol{x}, \boldsymbol{x}+\boldsymbol{e}_{j}\right)$. Adding all flows $\Psi_{\boldsymbol{x}, \boldsymbol{e}_{j}}$ we obtain a divergence free, unitary flow from $\partial_{-} B_{N}$ to $\mathcal{E}_{N}^{a}$ whose dissipated energy is easily estimated.

We start defining the paths. For $\boldsymbol{y} \in \mathbb{R}^{d}$, denote by $[\boldsymbol{y}]$ the vector whose $j$-th coordinate is $\left[\boldsymbol{y}_{j} N\right] / N$, where $[a]$ stands for the largest integer less than or equal to $a \in \mathbb{R}$. Fix $\boldsymbol{x} \in \partial_{-} B_{N}$. Denote by $\boldsymbol{x}(t)$ the solution of the ODE $\dot{\boldsymbol{x}}(t)=-\nabla F(\boldsymbol{x}(t))$ with initial condition $\boldsymbol{x}(0)=\boldsymbol{x}$. Since $[\boldsymbol{x}-\boldsymbol{z}] \cdot \boldsymbol{v}<0, \boldsymbol{x}(t)$ converges, as $t \rightarrow \infty$, to one of the local minima of $F$ in $W_{a}$. Let $T=\inf \left\{t>0: \boldsymbol{x}(t) \in W_{a}^{o}\right\}$, where $W_{a}^{o}$ is an open set whose closure is contained in $W_{a}^{\epsilon}$, the set introduced in (2.2). Let $\boldsymbol{y}^{0}=\boldsymbol{x}, \boldsymbol{y}^{1}, \ldots, \boldsymbol{y}^{m}$ be the sequence of points in $\Xi_{N}$ visited by the trajectory $[\boldsymbol{x}(t)]$, 
$0 \leq t \leq T$. If necessary, add points to this sequence in order to obtain a sequence $\boldsymbol{x}^{0}=\bar{x}, \boldsymbol{x}^{1}, \ldots, \boldsymbol{x}^{m^{\prime}}$ such that $\left\|\boldsymbol{x}^{k}-\boldsymbol{x}^{k+1}\right\|=N^{-1}$. Remove from this sequence the loops and denote by $n$ the length of the path. Since $F(\boldsymbol{x}(t))$ does not increase in time, and since for all $k$ there exists some $0 \leq t \leq T$ such that $\left\|\boldsymbol{x}^{k}-\boldsymbol{x}(t)\right\| \leq d / N$, there exists a finite constant $C_{0}$ such that

$$
F\left(\boldsymbol{x}^{k}\right) \leq F(\boldsymbol{x})+\frac{C_{0}}{N} \text { for all } 0 \leq k \leq n
$$

Fix a bond $\left(\boldsymbol{x}, \boldsymbol{x}+\boldsymbol{e}_{j}\right), \boldsymbol{x} \in \partial_{-} B_{N}, \boldsymbol{x}+\boldsymbol{e}_{j} \in B_{N}$. Define the flow $\Psi_{\boldsymbol{x}, \boldsymbol{e}_{j}}$ from $\boldsymbol{x}$ to $\mathcal{E}_{N}^{a}$ by $\Psi_{\boldsymbol{x}, \boldsymbol{e}_{j}}\left(\boldsymbol{x}^{k}, \boldsymbol{x}^{k+1}\right)=-\Psi\left(\boldsymbol{x}, \boldsymbol{x}+\boldsymbol{e}_{j}\right), 0 \leq k<n$. We claim that there exists a finite constant $C_{0}$ and a positive constant $c_{0}$ such that

$$
\left\|\Psi_{\boldsymbol{x}, \boldsymbol{e}_{j}}\right\|^{2} \leq C_{0} N Z_{N} e^{N F(\boldsymbol{z})} e^{-c_{0} N \varepsilon_{N}^{2}}
$$

The proof of this assertion is simple. Since $\Psi\left(\boldsymbol{x}, \boldsymbol{x}+\boldsymbol{e}_{j}\right)=\Phi\left(\boldsymbol{x}, \boldsymbol{x}+\boldsymbol{e}_{j}\right)$ is given by 4.3 , by 4.10,

$$
\left\|\Psi_{\boldsymbol{x}, \boldsymbol{e}_{j}}\right\|^{2} \leq C_{0} Z_{N} \sum_{k=0}^{n-1} e^{N F\left(\boldsymbol{x}^{k}\right)} \Phi\left(\boldsymbol{x}, \boldsymbol{x}+\boldsymbol{e}_{j}\right)^{2} \leq \frac{C_{0} Z_{N} n}{N^{d-1}} e^{N F(\boldsymbol{x})} e^{-N(\boldsymbol{y} \cdot \check{\mathbb{M}} \boldsymbol{y})}
$$

By a second order Taylor expansion, $\exp N\{F(\boldsymbol{x})-(\boldsymbol{y} \cdot \check{\mathbf{M}} \boldsymbol{y})\}$ is less than or equal to $C_{0} \exp \{N F(\boldsymbol{z})\} \exp \left\{-(1 / 2) \mu N \varepsilon_{N}^{2}\right\}$ because $N \varepsilon_{N}^{3} \rightarrow 0$ and $[\boldsymbol{x}-\boldsymbol{z}] \cdot \boldsymbol{v}<-\varepsilon_{N}$. This proves (4.11) because $n \leq\left|\Xi_{N}\right|$.

Let $\Psi=\sum_{\boldsymbol{x}, j} \Psi_{\boldsymbol{x}, \boldsymbol{e}_{j}}$, where the sum is carried over all $\boldsymbol{x}, j$ such that $\boldsymbol{x} \in \partial_{-} B_{N}$, $\boldsymbol{x}+\boldsymbol{e}_{j} \in B_{N} \cdot \Psi$ is a unitary, divergence free flow from $\partial_{-} B_{N}$ to $\varepsilon_{N}^{a}$. Moreover, by Schwarz inequality and by 4.11,

$$
\|\Psi\|^{2} \leq M \sum_{\boldsymbol{x}, j}\left\|\Psi_{\boldsymbol{x}, \boldsymbol{e}_{j}}\right\|^{2} \leq C_{0} N^{d+1} Z_{N} e^{N F(\boldsymbol{z})} e^{-c_{0} N \varepsilon_{N}^{2}}
$$

where $M$ represents the number of flows $\Psi_{\boldsymbol{x}, \boldsymbol{e}_{j}}$.

Choosing $\varepsilon_{N}$ appropriately and juxtaposing the flow just constructed with the one obtained in Section 1.D and a flow from $\partial_{+} Q_{N}$ to $\varepsilon_{N}^{b}$, similar to the one described in this section, yields a divergence free, unitary flow from $\mathcal{E}_{N}^{a}$ to $\mathcal{E}_{N}^{b}$, denoted by $\Phi_{z}$, such that

$$
\lim _{N \rightarrow \infty} \frac{(2 \pi N)^{d / 2}}{Z_{N}} \frac{1}{2 \pi N} e^{-N F(\boldsymbol{z})}\left\|\Phi_{z}\right\|^{2}=\frac{\sqrt{-\operatorname{det}[(\operatorname{Hess} F)(\boldsymbol{z})]}}{\mu(\boldsymbol{z})} .
$$

Step 2. Conclusion. Up to this point, for each saddle point $z$ separating $\mathcal{E}_{N}(A)$ from $\varepsilon_{N}\left(A^{c}\right)$ we constructed a divergence free, unitary flow $\Phi_{z}$ from $\varepsilon_{N}(A)$ to $\varepsilon_{N}\left(A^{c}\right)$ for which 4.12 holds. Denote the right hand side of 4.12 by $a(z)$ and observe that $F(\boldsymbol{z})$ is constant for $\boldsymbol{z} \in \mathfrak{S}(A)$.

Let $\Phi$ be a convex combination of the previous flows: $\Phi=\sum_{\boldsymbol{z} \in \mathfrak{S}(A)} \theta_{\boldsymbol{z}} \Phi_{\boldsymbol{z}}$, where $\theta_{\boldsymbol{z}} \geq 0, \sum_{\boldsymbol{z} \in \mathfrak{S}(A)} \theta_{\boldsymbol{z}}=1$. By construction, $\Phi$ is a flow from $\mathcal{E}_{N}(A)$ to $\mathcal{E}_{N}\left(A^{c}\right)$. On the other hand, since the saddle points are isolated and since the main contribution of the flow $\Phi_{z}$ occurs in a small neighborhood of $z$

$$
\limsup _{N \rightarrow \infty} \frac{(2 \pi N)^{d / 2}}{Z_{N}} \frac{1}{2 \pi N} e^{-N F(\boldsymbol{z})}\|\Phi\|^{2} \leq \sum_{\boldsymbol{z} \in \mathfrak{S}(A)} \theta_{\boldsymbol{z}}^{2} a(\boldsymbol{z})
$$


The optimal choice for $\theta$ is $\theta_{z}=a(\boldsymbol{z})^{-1} / \sum_{\boldsymbol{z}^{\prime}} a\left(\boldsymbol{z}^{\prime}\right)^{-1}$. With this choice the right hand side of the previous equation becomes $\left(\sum_{\boldsymbol{z} \in \mathfrak{S}(A)} a(\boldsymbol{z})^{-1}\right)^{-1}$. Proposition 4.1 follows from Thomson's principle and from the previous bound for the flow $\Phi$.

\section{Proof of Theorem 2.2}

Theorem 2.2 follows from Propositions 5.1 and 5.2 below. Throughout this section $1 \leq i \leq i_{0}$ and $1 \leq j \leq \ell_{i}$ are fixed and dropped from the notation.

Proposition 5.1. For every disjoint subsets $A, B$ of $S$,

$$
\operatorname{cap}_{N}\left(\mathcal{E}_{N}(A), \mathcal{E}_{N}(B)\right) \leq\left[1+o_{N}(1)\right] \frac{(2 \pi N)^{d / 2}}{Z_{N}} \frac{e^{-N H_{i}}}{2 \pi N} \operatorname{cap}_{\mathbb{G}}(A, B)
$$

The proof of this proposition is similar to the one of Proposition 3.1 up to Assertion 3.C. Denote by $\mathfrak{S}_{i, j}$ the set of all saddle points in $\Omega_{j}^{i}$, and recall the definition of the set $\mathfrak{U}_{N}$ introduced right after Assertion 3.C Let $\mathfrak{B}_{N}^{\boldsymbol{z}}=\mathfrak{U}_{N} \cap \mathcal{B}^{\boldsymbol{z}}$, $\boldsymbol{z} \in \mathfrak{S}_{i, j}, \nu_{N}=\mathcal{U}_{N} \backslash \cup_{\boldsymbol{z} \in \mathfrak{S}_{i, j}} \mathfrak{B}_{N}^{\boldsymbol{z}}$ so that

$$
\mathcal{U}_{N}=\mathcal{V}_{N} \cup \bigcup_{\boldsymbol{z} \in \mathfrak{S}_{i, j}} \mathfrak{B}_{N}^{\boldsymbol{z}}
$$

In contrast with Section 3 , we define a set $\mathfrak{B}_{N}^{\boldsymbol{z}}$ around each saddle point $\boldsymbol{z}$. By Assertion 3.C the set $\mathcal{V}_{N}$ is formed by several connected components separated by the sets $\mathfrak{B}_{N}^{\boldsymbol{z}}, \boldsymbol{z} \in \mathfrak{S}_{i, j}$. Let $\mathcal{V}_{N}^{a}$ be the connected component of $\mathcal{V}_{N}$ which contains a point in $W_{a}, a \in S$.

Fix two disjoint subsets $A, B$ of $S$ and denote by $V_{A, B}$ the equilibrium potential between $A$ and $B$ for the graph $\mathbb{G}$. Fix a saddle point $\boldsymbol{z} \in \mathfrak{S}_{i, j}$ and assume that $\boldsymbol{z} \in W_{a} \cap W_{b}$. Recall the definition of the function $V_{N}^{\boldsymbol{z}}$ introduced in (3.5) and assume without loss of generality that $\partial_{-} B_{N} \cap W_{a} \neq \varnothing$ so that $\partial_{+} B_{N} \cap \overline{W_{b}} \neq \varnothing$. Define $W_{N}^{\boldsymbol{z}}: \mathfrak{B}_{N}^{\boldsymbol{z}} \rightarrow[0,1]$ as

$$
W_{N}^{\boldsymbol{z}}(\boldsymbol{x})=V_{A, B}(a)+\left[V_{A, B}(b)-V_{A, B}(a)\right] V_{N}^{\boldsymbol{z}}(\boldsymbol{x}) .
$$

Let $V_{N}^{A, B}: \Xi_{N} \rightarrow[0,1]$ by

$$
V_{N}^{A, B}(\boldsymbol{x})= \begin{cases}V_{A, B}(a) & \boldsymbol{x} \in \mathcal{V}_{N}^{a} \\ W_{N}^{\boldsymbol{z}}(\boldsymbol{x}) & \boldsymbol{x} \in \mathfrak{B}_{N}^{\boldsymbol{z}} \\ (1 / 2) & \text { otherwise. }\end{cases}
$$

Assertion 5.A. Let $\varepsilon_{N}$ be a sequence such that $N \varepsilon_{N}^{3} \rightarrow 0, \exp \left\{-N \varepsilon_{N}^{2}\right\}$ converges to 0 faster than any polynomial. Then,

$$
\frac{Z_{N}}{(2 \pi N)^{d / 2}} 2 \pi N e^{N F(\boldsymbol{z})} D_{N}\left(V_{N}^{A, B}\right) \leq\left[1+o_{N}(1)\right] D_{\mathbb{G}}\left(V_{A, B}\right),
$$

where $D_{\mathbb{G}}\left(V_{A, B}\right)$ represents the Dirichlet form of $V_{A, B}$ with respect to the graph $\mathbb{G}$.

The proof of this assertion is similar to the one of Assertion 3.D. Proposition 5.1 follows from the last assertion and from the fact that $\operatorname{cap}_{\mathbb{G}}(A, B)=D_{\mathbb{G}}\left(V_{A, B}\right)$.

We conclude the section with the proof of the lower bound.

Proposition 5.2. For every disjoint subsets $A, B$ of $S$,

$$
\operatorname{cap}_{N}\left(\mathcal{E}_{N}(A), \mathcal{E}_{N}(B)\right) \geq\left[1+o_{N}(1)\right] \frac{(2 \pi N)^{d / 2}}{Z_{N}} \frac{e^{-N H_{i}}}{2 \pi N} \operatorname{cap}_{\mathbb{G}}(A, B)
$$


Proof. Fix two disjoint subsets $A, B$ of $S$. We construct below a divergence-free, unitary flow $\Psi$ from $\mathcal{E}_{N}(A)$ to $\mathcal{E}_{N}(B)$.

Recall that we denote by $V_{A, B}$ the equilibrium potential between $A$ and $B$ in the graph $\mathbb{G}$. Denote by $\varphi=\varphi_{A, B}$ the flow from $A$ to $B$ in the graph $\mathbb{G}$ given by $\varphi(a, b)=\boldsymbol{c}(a, b)\left[V_{A, B}(a)-V_{A, B}(b)\right] / \operatorname{cap}_{\mathbb{G}}(A, B)$, and observe that $\varphi(a, b)=0$ if $a$, $b$ belong to $A$ or if $a, b$ belong to $B$. By [16, Proposition 3.2.2],

$$
\frac{1}{\operatorname{cap}_{\mathbb{G}}(A, B)}=\frac{1}{2} \sum_{a, b \in S} \frac{1}{\boldsymbol{c}(a, b)} \varphi_{A, B}(a, b)^{2}=:\left\|\varphi_{A, B}\right\|^{2} .
$$

Assume first that each pair of wells has at most one saddle point separating them, that is, assume that the sets $W_{a} \cap W_{b}$ are either empty or singletons. In this case, each edge $(a, b)$ of the graph $\mathbb{G}$ corresponds to a unique saddle point $\boldsymbol{z}$.

Denote by $\Phi_{a, b}, a \neq b \in S, \boldsymbol{c}(a, b)>0$, the flow $\Phi_{\boldsymbol{z}}$ constructed just above 4.12 from $\varepsilon_{N}^{a}$ to $\mathcal{E}_{N}^{b}$, where $z \in W_{a} \cap W_{b}$ is the saddle point separating $W_{a}$ and $W_{b}$. Note that $\Phi_{a, b} \neq-\Phi_{b, a}$. We may assume that the flow $\Phi_{a, b}$ is a flow from $\boldsymbol{x}^{a}$ to $\boldsymbol{x}^{b}$, where $\boldsymbol{x}^{c}, c \in S$, are points in $\mathcal{E}_{N}^{c}$. Define the flow $\Psi$ by

$$
\Psi(\boldsymbol{x}, \boldsymbol{y})=\sum_{a, b} \varphi(a, b) \Phi_{a, b}(\boldsymbol{x}, \boldsymbol{y}), \quad \boldsymbol{x}, \boldsymbol{y} \in \Xi_{N},
$$

where the sum is carried out over all $a \neq b \in S$ such that $\varphi(a, b)>0$. We claim that $\Psi$ is a unitary, divergence-free flow from $\mathcal{E}_{N}(A)$ to $\mathcal{E}_{N}(B)$.

Clearly,

$$
\sum_{\boldsymbol{x} \in \mathcal{E}_{N}(A), \boldsymbol{y} \notin \mathcal{E}_{N}(A)} \Psi(\boldsymbol{x}, \boldsymbol{y})=\sum_{a, b} \varphi(a, b) \sum_{\boldsymbol{x} \in \mathcal{E}_{N}(A), \boldsymbol{y} \notin \mathcal{E}_{N}(A)} \Phi_{a, b}(\boldsymbol{x}, \boldsymbol{y}) .
$$

The flows $\Phi_{a, b}$ which cross $\varepsilon_{N}(A)$ are the ones starting or ending at $\mathcal{E}_{N}(A)$. Since, in addition, $\varphi(a, b)=0$ if $a, b \in A$, and $\varphi(a, b)<0$ if $a \notin A, b \in A$, the previous expression is equal to

$$
\sum_{a \in A, b \notin A} \varphi(a, b) \sum_{\boldsymbol{x} \in \mathcal{E}_{N}(A), \boldsymbol{y} \notin \mathcal{E}_{N}(A)} \Phi_{a, b}(\boldsymbol{x}, \boldsymbol{y})=\sum_{a \in A, b \notin A} \varphi(a, b),
$$

where the last identity follows from the fact that $\Phi_{a, b}$ is a unitary flow from $\varepsilon_{N}^{a}$ to $\mathcal{E}_{N}^{b}$. As $\varphi$ is a unitary flow from $A$ to $B$, the last sum is equal to 1 , proving that $\Psi$ is unitary.

To prove that $\Psi$ is divergence-free, fix a site $\boldsymbol{x} \notin\left\{\boldsymbol{x}^{c}: c \in A \cup B\right\}$. If $\boldsymbol{x} \notin\left\{\boldsymbol{x}^{c}\right.$ : $c \in S \backslash[A \cup B]\}, \Psi$ has no divergence at $\boldsymbol{x}$ because it is the convex combination of flows which have no divergence at $\boldsymbol{x}$. If $\boldsymbol{x}=\boldsymbol{x}^{c}, c \notin A \cup B$, the flows $\Phi_{a, b}, a$, $b \neq c$, have no divergence at $\boldsymbol{x}^{c}$, while the divergence of $\Phi_{a, c}\left(\operatorname{resp} . \Phi_{c, a}\right)$ at $\boldsymbol{x}^{c}$ is equal to -1 (resp. 1) because these flows are unitary and end (resp. start) at $\boldsymbol{x}^{c}$. Therefore, the divergence of $\Psi$ at $\boldsymbol{x}^{c}$ is equal to

$(\operatorname{div} \Psi)\left(\boldsymbol{x}^{c}\right)=\sum_{a, b} \varphi(a, b)\left(\operatorname{div} \Phi_{a, b}\right)\left(\boldsymbol{x}^{c}\right)=-\sum_{a: \varphi(a, c)>0} \varphi(a, c)+\sum_{a: \varphi(c, a)>0} \varphi(c, a)$.

Since $\varphi$ is a divergence-free flow in the graph $\mathbb{G}$, this sum vanishes, which proves that $\Psi$ is also divergence-free at $\boldsymbol{x}^{c}, c \in S \backslash[A \cup B]$.

We claim that the energy dissipated by the flow $\Psi$ is given by

$$
\|\Psi\|^{2}=\left[1+o_{N}(1)\right] \frac{Z_{N}}{(2 \pi N)^{d / 2}} 2 \pi N e^{N H_{i}} \frac{1}{\operatorname{cap}_{\mathbb{G}}(A, B)} .
$$


Indeed, by definition,

$$
\|\Psi\|^{2}=\sum_{j=1}^{d} \sum_{\boldsymbol{x}} \frac{1}{c\left(\boldsymbol{x}, \boldsymbol{x}+\boldsymbol{e}_{j}\right)} \Psi\left(\boldsymbol{x}, \boldsymbol{x}+\boldsymbol{e}_{j}\right)^{2},
$$

where the second sum is performed over all $\boldsymbol{x} \in \Xi_{N}$ such that $\boldsymbol{x}+\boldsymbol{e}_{j} \in \Xi_{N}$. By definition of the flow $\Psi$, the previous sum is equal to

$$
\begin{aligned}
& \sum_{a, b} \varphi(a, b)^{2} \sum_{j=1}^{d} \sum_{\boldsymbol{x}} \frac{1}{c\left(\boldsymbol{x}, \boldsymbol{x}+\boldsymbol{e}_{j}\right)} \Phi_{a, b}(\boldsymbol{x}, \boldsymbol{y})^{2} \\
& +\sum_{(a, b) \neq\left(a^{\prime}, b^{\prime}\right)} \varphi(a, b) \varphi\left(a^{\prime}, b^{\prime}\right) \sum_{j=1}^{d} \sum_{\boldsymbol{x}} \frac{1}{c\left(\boldsymbol{x}, \boldsymbol{x}+\boldsymbol{e}_{j}\right)} \Phi_{a, b}\left(\boldsymbol{x}, \boldsymbol{x}+\boldsymbol{e}_{j}\right) \Phi_{a^{\prime}, b^{\prime}}\left(\boldsymbol{x}, \boldsymbol{x}+\boldsymbol{e}_{j}\right) .
\end{aligned}
$$

By 4.9 , the first line is equal to

$$
\left[1+o_{N}(1)\right] \frac{Z_{N}}{(2 \pi N)^{d / 2}} 2 \pi N e^{N H_{i}} \sum_{a, b} \varphi(a, b)^{2} \frac{\sqrt{-\operatorname{det}\left[(\operatorname{Hess} F)\left(\boldsymbol{z}_{a, b}\right)\right]}}{\mu\left(\boldsymbol{z}_{a, b}\right)},
$$

where $\boldsymbol{z}_{a, b}$ stands for the saddle point in $W_{a} \cap W_{b}$ and $-\mu\left(\boldsymbol{z}_{a, b}\right)$ for the negative eigenvalue of $(\operatorname{Hess} F)\left(\boldsymbol{z}_{a, b}\right)$. By (2.4) and by (5.1), the previous sum is equal to

$$
\sum_{a, b} \frac{1}{\boldsymbol{c}(a, b)} \varphi(a, b)^{2}=\frac{1}{\operatorname{cap}_{\mathbb{G}}(A, B)} .
$$

We turn to the second line of (5.3). We have seen in the proof of Proposition 2.4 that the contribution of the bonds which do not belong to a mesoscopic neighborhood of the saddle point $\boldsymbol{z}_{a, b}$ to the total energy dissipated by the flow $\Phi_{a, b}$ is negligible. We may therefore restrict our attention in the second line of 5.3$)$ to the points $\boldsymbol{x}$ which belong to one of these neighborhoods. Since the flow $\Phi_{\boldsymbol{z}}$ vanishes in a neighborhood of a saddle point $\boldsymbol{z}^{\prime} \neq \boldsymbol{z}$, the product $\Phi_{a, b}(\boldsymbol{x}, \boldsymbol{y}) \Phi_{a^{\prime}, b^{\prime}}(\boldsymbol{x}, \boldsymbol{y})$ vanishes for all for $(a, b) \neq\left(a^{\prime}, b^{\prime}\right)$ and all $\boldsymbol{x}$ in a neighborhood of some saddle point $\boldsymbol{z}$. In particular, the second line of 5.3 is of order

$$
o_{N}(1) \frac{Z_{N}}{(2 \pi N)^{d / 2}} 2 \pi N e^{N H_{i}} .
$$

Assertion (5.2) follows from the estimates of the two lines of (5.3).

Since $\Psi$ is a divergence-free unitary flow from $\mathcal{E}^{N}(A)$ to $\mathcal{E}^{N}(B)$, by Thomson's principle, and by 5.2 ,

$$
\frac{1}{\operatorname{cap}_{N}\left(\mathcal{E}^{N}(A), \mathcal{E}^{N}(B)\right)} \leq\|\Psi\|^{2}=\left[1+o_{N}(1)\right] \frac{Z_{N}}{(2 \pi N)^{d / 2}} 2 \pi N e^{N H_{i}} \frac{1}{\operatorname{cap}_{\mathbb{G}}(A, B)} .
$$

This completes the proof of the proposition in the case where there is at most one saddle point between two wells.

In the general case, one has to change the definition of $\Psi$ as follows. For each $a, b \in S$ such that $\varphi(a, b)>0$, denote by $\boldsymbol{z}_{a, b}^{1}, \ldots, \boldsymbol{z}_{a, b}^{n}$ the set of saddle points between $W_{a}$ and $W_{b}: W_{a} \cap W_{b}=\left\{\boldsymbol{z}_{a, b}^{1}, \ldots, \boldsymbol{z}_{a, b}^{n}\right\}$, where $n=n_{a, b}$. Set

$$
\Psi=\sum_{a, b} \varphi(a, b) \sum_{k=1}^{n} \theta_{k}(a, b) \Phi_{\boldsymbol{z}_{a, b}^{k}},
$$


where the sum is carried out over all $a \neq b \in S$ such that $\varphi(a, b)>0$, where $\Phi_{\boldsymbol{z}_{a, b}^{k}}$ is the flow constructed just above 4.12 from $\varepsilon_{N}^{a}$ to $\varepsilon_{N}^{b}$ passing through the saddle point $\boldsymbol{z}_{a, b}^{k}$, and where

$$
\theta_{k}(a, b)=\frac{\mu\left(\boldsymbol{z}_{a, b}^{k}\right)}{\sqrt{-\operatorname{det}\left[(\operatorname{Hess} F)\left(\boldsymbol{z}_{a, b}^{k}\right)\right]}} \frac{1}{\boldsymbol{c}(a, b)} .
$$

Note that $\sum_{k} \theta_{k}(a, b)=1$. The arguments presented above for the case where there is at most one saddle point separating the wells can be easily adapted to the present case.

\section{Proof of Theorem 2.4}

According to [18, Theorem 5.1], Theorem 2.4 follows from Proposition 6.1 below.

Recall the notation introduced in Section 2, Fix $1 \leq i \leq i_{0}$ and $1 \leq j \leq \ell_{i}$, which are dropped out from the notation. Fix a connected component $\Omega=\Omega_{j}^{i}$, $1 \leq m \leq n=n_{i, j}$ and denote by $\mathcal{E}_{m, N}$ the union of the wells $\mathcal{E}_{N}^{a}, a \in S_{m}$, $\mathcal{E}_{m, N}=\cup_{a \in S_{m}} \varepsilon_{N}^{a}$. As $m$ is fixed throughout this section, it will sometimes be omitted from the notation.

Denote by $\left\{T_{m, N}(t): t \geq 0\right\}$ the additive functional

$$
T_{m, N}(t)=\int_{0}^{t} \mathbf{1}\left\{X_{N}(s) \in \mathcal{E}_{m, N}\right\} d s,
$$

and by $S_{m, N}(t)$ its generalized inverse: $S_{m, N}(t)=\sup \left\{s \geq 0: T_{m, N}(s) \leq t\right\}$. The time-change process $X_{N}^{m, \mathrm{~T}}(t):=X_{N}\left(S_{m, N}(t)\right)$ is called the trace process of $X_{N}(t)$ on $\varepsilon_{m, N}$. The process $X_{N}^{m, \mathrm{~T}}(t)$ is a $\mathcal{E}_{m, N}$-valued, continuous-time Markov chain. We refer to 2 for a summary of its properties.

Denote by $R_{N}^{m, T}(\boldsymbol{x}, \boldsymbol{y}), \boldsymbol{x}, \boldsymbol{y} \in \mathcal{E}_{N}$, the jump rates of the trace process. According to [2, Proposition 6.1],

$$
R_{N}^{m, \mathrm{~T}}(\boldsymbol{x}, \boldsymbol{y})=\lambda_{N}(\boldsymbol{x}) \mathbf{P}_{\boldsymbol{x}}\left[H_{\mathcal{E}_{m, N}}^{+}=H_{\boldsymbol{y}}\right], \quad \boldsymbol{x}, \boldsymbol{y} \in \mathcal{E}_{m, N}, \boldsymbol{x} \neq \boldsymbol{y} .
$$

Denote by $r_{N}^{m}(a, b)$ the average rate at which the trace process jumps from $\varepsilon_{N}^{a}$ to $\varepsilon_{N}^{b}, a, b \in S_{m}$ :

$$
r_{N}^{m}(a, b):=\frac{1}{\mu_{N}\left(\mathcal{E}_{N}^{a}\right)} \sum_{\boldsymbol{x} \in \mathcal{E}_{N}^{a}} \mu_{N}(\boldsymbol{x}) \sum_{\boldsymbol{y} \in \mathcal{E}_{N}^{b}} R_{N}^{m, \mathrm{~T}}(\boldsymbol{x}, \boldsymbol{y}) .
$$

Recall the definition of the projection $\Psi_{N}^{m}$ introduced in 2.7). Denote by $\boldsymbol{X}_{N}^{m, \mathrm{~T}}(t)$ the projection by $\Psi_{N}^{m}$ of the trace process $X_{N}^{m, \mathrm{~T}}(t), \boldsymbol{X}_{N}^{m, \mathrm{~T}}(t)=\Psi_{N}^{m}\left(X_{N}^{\mathrm{T}}(t)\right)$.

Proposition 6.1. Fix $1 \leq i \leq i_{0}, 1 \leq j \leq \ell_{i}, 1 \leq m \leq n_{i, j}, a \in S_{m}$ and $a$ sequence of configurations $\boldsymbol{x}_{N}$ in $\mathcal{E}_{N}^{a}$. Under $\mathbf{P}_{\boldsymbol{x}_{N}}$, the time re-scaled projection of the trace $\mathbb{X}_{N}^{m, \mathrm{~T}}(t)=\boldsymbol{X}_{N}^{m, \mathrm{~T}}\left(t \beta_{m}\right)$ converges in the Skorohod topology to a $S_{m}$ valued continuous-time Markov chain $\mathbb{X}^{m}(t)$ whose jump rates are given by (2.9). Moreover, in the time scale $\beta_{m}$, the time spent by the original chain $X_{N}(t)$ outside $\mathcal{E}_{m, N}$ is negligible: for all $t>0$,

$$
\lim _{N \rightarrow \infty} \mathbf{E}_{\boldsymbol{x}_{N}}\left[\int_{0}^{t} \mathbf{1}\left\{X_{N}\left(s \beta_{m}\right) \notin \mathcal{E}_{m, N}\right\} d s\right]=0 .
$$


Proof. By [2, Theorem 2.7], the first assertion of the proposition follows by Lemmata 6.2 and 6.3 below. We turn to the proof of the second assertion of the proposition.

Fix $\delta>0$ such that $\delta<H_{i+1}-H_{i}$ and let $\widetilde{\Omega}_{\delta}^{i}=\left\{\boldsymbol{x} \in \Xi: F(\boldsymbol{x}) \leq H_{i}+\delta\right\}$. Denote by $\widetilde{\Omega}_{\delta}=\widetilde{\Omega}_{\delta}^{i, j}$ the connected component which contains $\Omega_{j}^{i}$ and let $A_{N}=\widetilde{\Omega}_{\delta} \cap \Xi_{N}$.

By the large deviations principle for the chain $X_{N}(t)$, for every $T>0$ and every sequence $\boldsymbol{x}_{N} \in \mathcal{E}_{1, N}$,

$$
\lim _{N \rightarrow \infty} \mathbf{P}_{\boldsymbol{x}_{N}}\left[H_{A_{N}^{c}} \leq T \beta_{m}\right]=0 .
$$

This statement can be proved as Theorem 4.2 of Chapter 4, or Theorem 6.2 of Chapter 6 in [14. It is therefore enough to prove 6.2 for the chain $X_{N}(t)$ reflected at $A_{N}$, the chain obtained by removing all jumps between $A_{N}$ and $A_{N}^{c}$.

Denote the reflected chain by $\tilde{X}_{N}(t)$, by $\tilde{\mu}_{N}$ its stationary state, and by $\tilde{\mathbf{P}}_{\boldsymbol{x}}$ the measure on the path space $D\left(\mathbb{R}_{+}, A_{N}\right)$ induced by the chain $\tilde{X}_{N}(t)$ starting from $\boldsymbol{x} \in A_{N}$. Expectation with respect to $\tilde{\mathbf{P}}_{\boldsymbol{x}}$ is represented by $\tilde{\mathbf{E}}_{\boldsymbol{x}}$. We have to prove 6.2 with $X_{N}(t), \mathbf{E}_{\boldsymbol{x}_{N}}$ replaced by $\tilde{X}_{N}(t), \tilde{\mathbf{E}}_{\boldsymbol{x}}$, respectively. Equation 6.2 with these replacements is represented as $(6.2 *)$.

Let $\Delta_{m, N}=A_{N} \backslash \varepsilon_{m, N}$. By definition of the sets $\mathcal{E}_{N}^{a}$, for $a \in S_{1}, \tilde{\mu}_{N}\left(\Delta_{1, N}\right) / \tilde{\mu}_{N}\left(\mathcal{E}_{N}^{a}\right)$ is at most of the order $\exp \left\{-N\left(\theta_{1}-\epsilon\right)\right\}$, where $\epsilon$ has been introduced right before 2.2. For each fixed $1<m \leq n, a \in S_{m}, \tilde{\mu}_{N}\left(\Delta_{m, N}\right) / \tilde{\mu}_{N}\left(\varepsilon_{N}^{a}\right)$ is at most of the order $\exp \left\{-N\left(\theta_{m}-\theta_{m-1}-\epsilon\right)\right\}$. Therefore, for every $1 \leq m \leq n, a \in S_{m}$,

$$
\lim _{N \rightarrow \infty} \frac{\tilde{\mu}_{N}\left(\Delta_{m, N}\right)}{\tilde{\mu}_{N}\left(\mathcal{E}_{N}^{a}\right)}=0 \text {. }
$$

Fix $1 \leq m<n, a \in S_{m+1}$ and a sequence $\boldsymbol{x}_{N} \in \mathcal{E}_{N}^{a}$. By the large deviations principle for the chain $\tilde{X}_{N}(t)$, for every $T>0$,

$$
\lim _{N \rightarrow \infty} \tilde{\mathbf{P}}_{\boldsymbol{x}_{N}}\left[H_{\left(\varepsilon_{N}^{a}\right)^{c}} \leq T \beta_{m}\right]=0 .
$$

By [2, Theorem 2.7], assertion (6.2*) follows from the first part of this proposition and from $(6.3)$, 6.4 , which concludes the proof.

Recall that we denoted by $\left\{\boldsymbol{m}_{a, 1}, \ldots, \boldsymbol{m}_{a, q}\right\}, q=q_{a}$, the deepest local minima of $F$ which belong to $W_{a}$.

Lemma 6.2. Under the hypotheses of Proposition 6.1, for every $a \in S_{m}$,

$$
\lim _{N \rightarrow \infty} \sup _{\boldsymbol{y} \in \mathcal{E}_{N}^{a}} \frac{\operatorname{cap}_{N}\left(\mathcal{E}_{N}^{a}, \cup_{b \in S_{m}, b \neq a} \mathcal{E}_{N}^{b}\right)}{\operatorname{cap}_{N}\left(\{\boldsymbol{y}\},\left\{\boldsymbol{m}_{a, 1}\right\}\right)}=0 .
$$

Proof. Fix $a \in S_{m}, \boldsymbol{y} \in \mathcal{E}_{N}^{a}$. We estimate $\operatorname{cap}_{N}\left(\left\{\boldsymbol{y},\left\{\boldsymbol{m}_{a, 1}\right\}\right)\right.$ through Thomson's principle. Let $\left(\boldsymbol{y}=\boldsymbol{x}_{0}, \boldsymbol{x}_{1}, \ldots, \boldsymbol{x}_{m}=\boldsymbol{m}_{a, 1}\right)$ be a path $\gamma$ from $\boldsymbol{y}$ to $\boldsymbol{m}_{a, 1}$ so that $\left\|\boldsymbol{x}_{j}-\boldsymbol{x}_{j+1}\right\|=1 / N$. By Thomson's principle,

$$
\frac{1}{\operatorname{cap}_{N}\left(\{\boldsymbol{y}\},\left\{\boldsymbol{m}_{a, 1}\right\}\right)} \leq \sum_{j=0}^{m-1} \frac{1}{\mu_{N}\left(\boldsymbol{x}_{j}\right) R_{N}\left(\boldsymbol{x}_{j}, \boldsymbol{x}_{j+1}\right)} .
$$

In view of the explicit formulas for the measure $\mu_{N}$ and the rates $R_{N}$, there exists a finite constant $C_{0}$ such that

$$
\frac{1}{\mu_{N}\left(\boldsymbol{x}_{j}\right) R_{N}\left(\boldsymbol{x}_{j}, \boldsymbol{x}_{j+1}\right)} \leq C_{0} Z_{N} e^{N F\left(\boldsymbol{x}_{j}\right)} .
$$


It follows from the definition 2.2 of the set $W_{a}^{\epsilon}$ that the path $\gamma$ can be chosen in such a way that $F\left(\boldsymbol{x}_{j}\right) \leq H_{i}-\epsilon$. The previous sum is thus bounded above by $C_{0} Z_{N} N \exp N\left\{H_{i}-\epsilon\right\}$, where $N$ has been introduced to take care of the length of the path. This estimate is uniform over $\boldsymbol{y} \in \mathcal{E}_{N}^{a}$. To conclude the proof of the lemma, it remains to recall the assertion of Theorem 2.2 .

Lemma 6.3. Under the hypotheses of Proposition 6.1, for every $a, b \in S_{m}$,

$$
\lim _{N \rightarrow \infty} \beta_{m} r_{N}^{m}(a, b)=\boldsymbol{r}_{m}(a, b)
$$

where the rates $\boldsymbol{r}_{m}(a, b)$ are given by 2.9 .

Proof. By 2, Lemma 6.8],

$$
\begin{array}{r}
r_{N}^{m}(a, b)=\frac{1}{2} \frac{1}{\mu_{N}\left(\mathcal{E}_{N}^{a}\right)}\left\{\operatorname{cap}_{N}\left(\mathcal{E}_{N}^{a}, \mathcal{E}_{m, N} \backslash \mathcal{E}_{N}^{a}\right)+\operatorname{cap}_{N}\left(\mathcal{E}_{N}^{b}, \mathcal{E}_{m, N} \backslash \mathcal{E}_{N}^{b}\right)\right. \\
\left.-\operatorname{cap}_{N}\left(\varepsilon_{N}^{a} \cup \mathcal{E}_{N}^{b}, \varepsilon_{m, N} \backslash\left[\mathcal{E}_{N}^{a} \cup \mathcal{E}_{N}^{b}\right]\right)\right\} .
\end{array}
$$

By (2.3),

$$
\mu_{N}\left(\varepsilon_{N}^{a}\right)=\left[1+o_{N}(1)\right] \frac{(2 \pi N)^{d / 2}}{Z_{N}} \frac{e^{-N H_{i}}}{2 \pi N} \beta_{m} \boldsymbol{\mu}(a) .
$$

The assertion of the lemma follows from this equation, Theorem 2.2 and the definition of $\boldsymbol{c}_{m}$ given just above 2.9 .

We conclude this section with a calculation which provides an estimation for the measure of the wells. Denote by $\boldsymbol{m}^{1}, \ldots, \boldsymbol{m}^{r}$ the global minima of $F$ on $\Xi$. We claim that

$$
\lim _{N \rightarrow \infty} \frac{e^{N F\left(\boldsymbol{m}^{1}\right)}}{(2 \pi N)^{d / 2}} Z_{N}=\sum_{k=1}^{r} \frac{1}{\sqrt{\operatorname{det}\left[(\operatorname{Hess} F)\left(\boldsymbol{m}^{k}\right)\right]}} .
$$

A similar argument yields (2.3).

Indeed, fix a sequence $\varepsilon_{N}$ such that $\lim _{N \rightarrow \infty} N \varepsilon_{N}^{3}=0$ and for which $\exp \left\{-N \varepsilon_{N}^{2}\right\}$ vanishes faster than any polynomial. Fix $1 \leq k \leq r$ and denote by $\boldsymbol{w}^{1}, \ldots, \boldsymbol{w}^{d}$ the eigenvectors of $(\operatorname{Hess} F)\left(\boldsymbol{m}^{k}\right)$ and by $0<\lambda_{1} \leq \cdots \leq \lambda_{d}$ the eigenvalues. Consider the neighborhood $B_{N}$ of $\boldsymbol{m}^{k}$ defined by

$$
B_{N}=\left\{\boldsymbol{x} \in \Xi_{N}:\left|\left(\boldsymbol{x}-\boldsymbol{m}^{k}\right) \cdot \boldsymbol{w}^{i}\right| \leq \varepsilon_{N}, 1 \leq i \leq d\right\} .
$$

It follows from the assumptions on $\varepsilon_{N}$ and on $F$, from a second-order Taylor expansion of $F$ around $\boldsymbol{m}^{k}$, and from a simple calculation that

$$
\lim _{N \rightarrow \infty} \frac{e^{N F\left(\boldsymbol{m}^{1}\right)}}{(2 \pi N)^{d / 2}} \sum_{\boldsymbol{x} \in B_{N}} e^{-N F(\boldsymbol{x})}=\frac{1}{\sqrt{\operatorname{det}\left[(\operatorname{Hess} F)\left(\boldsymbol{m}^{k}\right)\right]}} .
$$

Denote by $B_{N}^{(2)}$ the neighborhood of $\boldsymbol{m}^{k}$ defined by

$$
B_{N}^{(2)}=\left\{\boldsymbol{x} \in \Xi_{N}:\left\|\boldsymbol{x}-\boldsymbol{m}^{k}\right\| \leq \lambda_{1} /\left(4 C_{1}\right)\right\},
$$

where $C_{1}$ is the Lipschitz constant introduced in assumption (H1). Clearly, on $B_{N}^{(2)}, F(\boldsymbol{x})-F\left(\boldsymbol{m}^{1}\right) \geq(1 / 2) \lambda_{1}\left\|\boldsymbol{x}-\boldsymbol{m}^{k}\right\|^{2}-C_{1}\left\|\boldsymbol{x}-\boldsymbol{m}^{k}\right\|^{3} \geq\left(\lambda_{1} / 4\right)\left\|\boldsymbol{x}-\boldsymbol{m}^{k}\right\|^{2}$. Therefore, as $\left\|\boldsymbol{x}-\boldsymbol{m}^{k}\right\|^{2} \geq \varepsilon_{N}^{2}$ on $B_{N}^{c}$ and as $N \varepsilon_{N}^{2} \rightarrow \infty$,

$$
\lim _{N \rightarrow \infty} \frac{e^{N F\left(\boldsymbol{m}^{1}\right)}}{(2 \pi N)^{d / 2}} \sum_{\boldsymbol{x} \in B_{N}^{(2)} \backslash B_{N}} e^{-N F(\boldsymbol{x})}=0 .
$$


On the complement of the union of all $B_{N}^{(2)}$-neighborhoods of the minima $\boldsymbol{m}^{k}$, $F(\boldsymbol{x})-F\left(\boldsymbol{m}^{1}\right) \geq \delta$ for some $\delta>0$. In particular the contribution to $Z_{N}$ of the sum over this set is negligible. Putting together all previous estimates we obtain (6.5).

\section{Proof of Theorem 2.7}

We prove in this section Theorem 2.7. Recall the notation introduced in Subsection 2D. Hereafter, $C_{0}$ represents a finite constant independent of $N$ which may change from line to line. We start with some preliminary results.

Lemma 7.1. Fix $1 \leq i \leq i_{0}, 1 \leq j \leq \ell_{i}$, and $a \in S=\left\{1, \ldots \ell_{j}^{i}\right\}$. Let $\mathcal{B}_{a}=$ $\cup_{\boldsymbol{z} \in \mathfrak{S}_{a}} \mathcal{D}_{\boldsymbol{z}}$. For any sequence $\left\{\boldsymbol{x}_{N}: N \geq 1\right\}, \boldsymbol{x}_{N} \in \mathcal{E}_{N}^{a}$,

$$
\lim _{N \rightarrow \infty} \mathbf{P}_{\boldsymbol{x}_{N}}\left[H_{\mathcal{D}_{a}}=H_{\mathcal{B}_{a}}\right]=1 \text {. }
$$

Proof. By [4, Lemma 4.3],

$$
\mathbf{P}_{\boldsymbol{x}_{N}}\left[H_{\mathcal{D}_{a}}<H_{\mathcal{B}_{a}}\right] \leq \frac{\operatorname{cap}_{N}\left(\boldsymbol{x}_{N}, \mathcal{D}_{a} \backslash \mathcal{B}_{a}\right)}{\operatorname{cap}_{N}\left(\boldsymbol{x}_{N}, \mathcal{D}_{a}\right)} .
$$

Let $V: \Xi_{N} \rightarrow[0,1]$ be the indicator of the set $\mathcal{D}_{a} \backslash \mathcal{B}_{a}$. By the Dirichlet principle and a straightforward computation, $\operatorname{cap}_{N}\left(\boldsymbol{x}_{N}, \mathcal{D}_{a} \backslash \mathcal{B}_{a}\right) \leq D_{N}(V) \leq$ $C_{0} Z_{N}^{-1} N^{d} \exp \left\{-N\left(H_{i}+\delta_{N}\right)\right\}$. On the other hand, it is not difficult to construct a divergence-free, unitary flow $\Phi$ from $\mathcal{B}_{a}$ to $\boldsymbol{x}_{N}$, similar to the one presented in the proof of Lemma 6.2. such that $\|\Phi\|^{2} \leq C_{0} Z_{N} N \exp \left\{-N H_{i}\right\}$. Therefore, by Thomson's principle, $\operatorname{cap}_{N}\left(\boldsymbol{x}_{N}, \mathcal{D}_{a}\right)^{-1} \leq C_{0} Z_{N} N \exp \left\{-N H_{i}\right\}$, which proves the lemma in view of the definition of the sequence $\delta_{N}$.

Fix $\boldsymbol{z} \in \mathfrak{S}_{a}$ and recall that we denote by $\boldsymbol{v}=\boldsymbol{w}^{1}, \boldsymbol{w}^{j}, 2 \leq j \leq d$, a basis of eigenvectors of Hess $F(\boldsymbol{z})$, where $\boldsymbol{v}$ is the one associated to the unique negative eigenvalue $-\mu$. Let $B_{N}=B_{N}^{\boldsymbol{z}}$ be a mesoscopic neighborhood of $\boldsymbol{z}$ :

$$
B_{N}=\left\{\boldsymbol{x} \in \Xi_{N}:|(\boldsymbol{x}-\boldsymbol{z}) \cdot \boldsymbol{v}| \leq a \varepsilon_{N}, \max _{2 \leq j \leq d}\left|(\boldsymbol{x}-\boldsymbol{z}) \cdot \boldsymbol{w}^{j}\right| \leq \varepsilon_{N}\right\},
$$

where $a=\max \left\{1, \mu^{-1}\left(1+\sum_{2 \leq j \leq d} \lambda_{j}\right)\right\}$, and $\varepsilon_{N}$ is a sequence of positive numbers such that $N^{-2} \ll \varepsilon_{N}^{4} \ll N^{-3 / 2}, \varepsilon_{N}^{2} \gg \delta_{N}$. The sets $D_{\boldsymbol{z}}, \boldsymbol{z} \in \mathfrak{S}_{a}$, are contained in $B_{N}$ because, by 2.11 and 2.12,

$$
\sup _{\boldsymbol{x} \in D_{\boldsymbol{z}}}\|\boldsymbol{x}-\boldsymbol{z}\|^{2} \leq\left(4 / \lambda_{2}\right) \delta_{N}
$$

Recall from (3.3) the definition of the outer boundary $\partial B_{N}$ of $B_{N}$, and let $\partial_{-} B_{N}$, $\partial_{+} B_{N}$ be the pieces of the outer boundary of $B_{N}$ defined by

$$
\begin{gathered}
\partial_{-} B_{N}=\left\{\boldsymbol{x} \in \partial B_{N}:(\boldsymbol{x}-\boldsymbol{z}) \cdot \boldsymbol{v}<-a \varepsilon_{N}\right\}, \\
\partial_{+} B_{N}=\left\{\boldsymbol{x} \in \partial B_{N}:(\boldsymbol{x}-\boldsymbol{z}) \cdot \boldsymbol{v}>a \varepsilon_{N}\right\} .
\end{gathered}
$$

A Taylor expansion of $F$ around $z$ shows that

$$
\max _{\boldsymbol{x} \in \partial_{-} B_{N} \cup \partial_{+} B_{N}} F(\boldsymbol{x}) \leq H_{i}-\frac{1}{2} \varepsilon_{N}^{2}\left(1+O\left(\varepsilon_{N}\right)\right) .
$$

Denote by $H_{N}$ the hitting time of the boundary $\partial B_{N}$, and by $H_{N}^{ \pm}$the hitting time of the sets $\partial_{ \pm} B_{N}$. 
Proposition 7.2. For every $\boldsymbol{z} \in \mathfrak{S}_{a}$,

$$
\lim _{N \rightarrow \infty} \max _{\boldsymbol{x} \in \mathcal{D}_{\boldsymbol{z}}}\left|\mathbf{P}_{\boldsymbol{x}}\left[H_{N}=H_{N}^{ \pm}\right]-\frac{1}{2}\right|=0 .
$$

Corollary 7.3. Let $\left\{\boldsymbol{x}_{N}^{c}: N \geq 1\right\}, c \in S$, be a sequence of points in $\mathcal{E}_{N}^{c}$ and let $\hat{S}=\hat{S}_{N}=\left\{\boldsymbol{x}_{N}^{c}: c \in S\right\}$. Fix $a \neq b \in S$ and $\boldsymbol{z} \in \mathfrak{S}_{a, b}$. Then,

$$
\lim _{N \rightarrow \infty} \max _{\boldsymbol{x} \in \mathcal{D}_{\boldsymbol{z}}}\left|\mathbf{P}_{\boldsymbol{x}}\left[H_{\hat{S}}=H_{\boldsymbol{x}_{N}^{c}}\right]-q(c)\right|=0,
$$

where $q(a)=q(b)=1 / 2$ and $q(c)=0$ for $c \in S \backslash\{a, b\}$.

Proof. Fix $a \neq b \in S, c \in S, \boldsymbol{z} \in \mathfrak{S}_{a, b}$ and $\boldsymbol{x} \in \mathcal{D}_{\boldsymbol{z}}$. Since $H_{N} \leq H_{\hat{S}}$, by the strong Markov property,

$$
\mathbf{P}_{\boldsymbol{x}}\left[H_{\hat{S}}=H_{\boldsymbol{x}_{N}^{c}}\right]=\mathbf{E}_{\boldsymbol{x}}\left[\mathbf{P}_{X_{N}\left(H_{N}\right)}\left[H_{\hat{S}}=H_{\boldsymbol{x}_{N}^{c}}\right]\right] .
$$

By the proposition, the previous expression is equal to

$$
\sum_{\boldsymbol{y} \in \partial_{ \pm} B_{N}} \mathbf{P}_{\boldsymbol{x}}\left[X_{N}\left(H_{N}\right)=\boldsymbol{y}\right] \mathbf{P}_{\boldsymbol{y}}\left[H_{\hat{S}}=H_{\boldsymbol{x}_{N}^{c}}\right]+R_{N}(\boldsymbol{x})
$$

where $\lim _{N \rightarrow \infty} \max _{\boldsymbol{x} \in \mathcal{D}_{\boldsymbol{z}}}\left|R_{N}(\boldsymbol{x})\right|=0$.

Let $\boldsymbol{x}(t), 0 \leq t \leq 1$, be a continuous path from $\boldsymbol{x}_{N}^{a}$ to $\boldsymbol{x}_{N}^{b}$ for which there exists $0<t_{0}<1$ such that $F(\boldsymbol{x}(t))<H_{i}$ for all $t \neq t_{0}$ and $\boldsymbol{x}\left(t_{0}\right)=\boldsymbol{z}$. Assume that this path crosses $B_{N}$ only at $\partial_{ \pm} B_{N}$ and assume, without loss of generality, that it crosses $\partial_{-} B_{N}$ before $\partial_{+} B_{N}$. In this case, an argument similar to the one presented in the proof of Lemma 7.1 yields that

$$
\lim _{N \rightarrow \infty} \min _{\boldsymbol{y} \in \partial_{+} B_{N}} \mathbf{P}_{\boldsymbol{y}}\left[H_{\hat{S}}=H_{\boldsymbol{x}_{N}^{b}}\right]=1, \quad \lim _{N \rightarrow \infty} \min _{\boldsymbol{y} \in \partial_{-} B_{N}} \mathbf{P}_{\boldsymbol{y}}\left[H_{\hat{S}}=H_{\boldsymbol{x}_{N}^{a}}\right]=1 .
$$

In the proof of this assertion, instead of using an indicator function to bound from above the capacity, as we did in the proof of Lemma 7.1, we use the function constructed in Section 3. Note also that if the continuous path from $\boldsymbol{x}_{N}^{a}$ to $\boldsymbol{x}_{N}^{b}$ crosses first $\partial_{+} B_{N}$ and then $\partial_{-} B_{N}$, one has to interchange $a$ and $b$ in the previous displayed formula.

Up to this point we showed that

$\mathbf{P}_{\boldsymbol{x}}\left[H_{\hat{S}}=H_{\boldsymbol{x}_{N}^{c}}\right]=\mathbf{P}_{\boldsymbol{x}}\left[H_{N}=H_{N}^{-}\right] \mathbf{1}\{c=a\}+\mathbf{P}_{\boldsymbol{x}}\left[H_{N}=H_{N}^{+}\right] \mathbf{1}\{c=b\}+R_{N}^{\prime}(\boldsymbol{x})$, where $R_{N}^{\prime}(\boldsymbol{x})$ is a new sequence with the same properties as the previous one. To complete the proof it remains to recall the statement of the proposition.

The proof of Proposition 7.2 is based on the fact that in a neighborhood of radius $N^{-1 / 2}$ around a saddle point $\boldsymbol{z}$ the re-scaled chain $\sqrt{N} X_{N}(t N)$ behaves as a diffusion. More precisely, let $g: \mathbb{R}^{d} \rightarrow \mathbb{R}$ be a three times continuously differentiable function and let $G(\boldsymbol{x})=g\left(\sqrt{N}(\boldsymbol{x}-\boldsymbol{z}) \cdot \boldsymbol{w}^{1}, \ldots, \sqrt{N}(\boldsymbol{x}-\boldsymbol{z}) \cdot \boldsymbol{w}^{d}\right)$. A Taylor expansion of the potential $F$ around $\boldsymbol{z}$ gives that for $\boldsymbol{x} \in B_{N}$,

$$
\left(L_{N} G\right)(\boldsymbol{x})=\frac{1}{N} \sum_{j=1}^{d}\left\{-\lambda_{j} \boldsymbol{u}_{j}\left(\partial_{\boldsymbol{x}_{j}} g\right)(\boldsymbol{u})+\left(\partial_{\boldsymbol{x}_{j}}^{2} g\right)(\boldsymbol{u})\right\}+\frac{R_{N}}{N},
$$

where $\boldsymbol{u}_{j}=\sqrt{N}(\boldsymbol{x}-\boldsymbol{z}) \cdot \boldsymbol{w}^{j}$, and $R_{N}$ is an error term satisfying

$$
\left|R_{N}\right| \leq C_{0} N \varepsilon_{N}^{2}\left\{\frac{C_{1}(g)}{\sqrt{N}}+\frac{C_{2}(g)}{N}\right\}+C_{0} \frac{C_{3}(g)}{\sqrt{N}} .
$$


In this formula, $C_{1}(g)=\max _{1 \leq j \leq d} \sup _{\boldsymbol{u},\|\boldsymbol{u}\| \leq a \sqrt{N} \varepsilon_{N}}\left|\left(\partial_{\boldsymbol{x}_{j}} g\right)(\boldsymbol{u})\right|$, with a similar definition for $C_{2}(g)$ and $C_{3}(g)$, replacing first derivates by second and thirds.

Identity (7.4) asserts that the process $\left(\sqrt{N}\left(X_{N}(t N)-\boldsymbol{z}\right) \cdot \boldsymbol{w}^{1}, \ldots, \sqrt{N}\left(X_{N}(t N)-\right.\right.$ $\left.\boldsymbol{z}) \cdot \boldsymbol{w}^{d}\right)$ is close to a diffusion whose coordinates evolve independently. The first coordinate has a drift towards $\pm \infty$ proportional to its distance to the origin, while the other coordinates are Ornstein-Uhlenbeck processes.

Lemma 7.4. There exists a finite constant $C_{0}$ such that for every $\boldsymbol{z} \in \mathfrak{S}_{a}$,

$$
\max _{\boldsymbol{x} \in \mathcal{D}_{\boldsymbol{z}}} \mathbf{E}_{\boldsymbol{x}}\left[H_{N}\right] \leq C_{0} N^{3 / 2} \varepsilon_{N}
$$

Proof. Let $g: \mathbb{R} \rightarrow \mathbb{R}$ be given by $g(x)=\int_{0}^{x} \exp \left\{-\mu y^{2} / 2\right\} \int_{0}^{y} \exp \left\{\mu z^{2} / 2\right\} d z d y$. It is clear that $g$ solves the differential equation $\mu x g^{\prime}(x)+g^{\prime \prime}(x)=1, x \in \mathbb{R}$. By Dynkin's formula, for every $t>0, \boldsymbol{x} \in \mathcal{D}_{\boldsymbol{z}}$,

$$
\mathbf{E}_{\boldsymbol{x}}\left[G\left(X_{N}\left(t \wedge H_{N}\right)\right)-G(\boldsymbol{x})-\int_{0}^{t \wedge H_{N}}\left(L_{N} G\right)\left(X_{N}(s)\right) d s\right]=0,
$$

where $G(\boldsymbol{x})=g\left(N^{1 / 2}[\boldsymbol{x}-\boldsymbol{z}] \cdot \boldsymbol{v}\right)$. By 7.4 and since $\left|g^{\prime}(x)\right| \leq C_{0},\left|g^{\prime \prime}(x)\right| \leq$ $C_{0}|x|,\left|g^{\prime \prime \prime}(x)\right| \leq C_{0} x^{2}$, on $B_{N}, N\left(L_{N} G\right)(x)-1$ is absolutely bounded by $C_{0} \sqrt{N} \varepsilon_{N}^{2}$. Therefore,

$$
\left(1-C_{0} \sqrt{N} \varepsilon_{N}^{2}\right) \mathbf{E}_{\boldsymbol{x}}\left[t \wedge H_{N}\right] \leq N \mathbf{E}_{\boldsymbol{x}}\left[G\left(X_{N}\left(t \wedge H_{N}\right)\right)\right] .
$$

Since $|g(x)| \leq C_{0}|x|, \sup _{\boldsymbol{x} \in B_{N}}|G(\boldsymbol{x})| \leq C_{0} \sqrt{N} \varepsilon_{N}$. To complete the proof of the lemma, it remains to observe that $\sqrt{N} \varepsilon_{N}^{2} \rightarrow 0$ and to let $t \uparrow \infty$.

Lemma 7.5. For every $z \in \mathfrak{S}_{a}$,

$$
\lim _{N \rightarrow \infty} \max _{\boldsymbol{x} \in \mathcal{D}_{\boldsymbol{z}}} \mathbf{P}_{\boldsymbol{x}}\left[H_{N}<H_{N}^{+} \wedge H_{N}^{-}\right]=0 .
$$

Proof. The proof is similar to the one of the previous lemma. Fix $2 \leq j \leq d$ and let $g: \mathbb{R} \rightarrow \mathbb{R}$ be given by $g(x)=x^{2}$. By Dynkin's formula, for every $t>0, \boldsymbol{x} \in \mathcal{D}_{\boldsymbol{z}}$, 7.5. holds with $G(\boldsymbol{x})=g\left(N^{1 / 2}[\boldsymbol{x}-\boldsymbol{z}] \cdot \boldsymbol{w}^{j}\right)$. By 7.4$),\left(L_{N} G\right)(\boldsymbol{x}) \leq\left(2+R_{N}\right) / N$ and $R_{N} / N \leq C_{0} \varepsilon_{N}^{3}$. Therefore, letting $t \uparrow \infty$, by Lemma 7.4 we get that

$$
\begin{aligned}
\mathbf{E}_{\boldsymbol{x}}\left[G\left(X_{N}\left(H_{N}\right)\right)\right] & \leq G(\boldsymbol{x})+\left(\frac{2}{N}+C_{0} \varepsilon_{N}^{3}\right) \mathbf{E}_{\boldsymbol{x}}\left[H_{N}\right] \\
& \leq G(\boldsymbol{x})+C_{0}\left(\frac{1}{N}+C_{0} \varepsilon_{N}^{3}\right) N^{3 / 2} \varepsilon_{N} .
\end{aligned}
$$

The event $\mathcal{A}_{N}=\left\{\left|\left(X_{N}\left(H_{N}\right)-\boldsymbol{z}\right) \cdot \boldsymbol{w}^{j}\right|>\varepsilon_{N}\right\}$ corresponds to the event that the process $X_{N}(t)$ reaches the boundary of $B_{N}$ by hitting the set $\left\{\boldsymbol{x} \in B_{N}\right.$ : $\left.[\boldsymbol{x}-\boldsymbol{z}] \cdot \boldsymbol{w}^{j}= \pm \varepsilon_{N}\right\}$. On this event the function $G$ is equal to $N \varepsilon_{N}^{2}$. Since $G$ is nonnegative,

$$
N \varepsilon_{N}^{2} \mathbf{P}_{\boldsymbol{x}}\left[\mathcal{A}_{N}\right] \leq \mathbf{E}_{\boldsymbol{x}}\left[G\left(X_{N}\left(H_{N}\right)\right)\right]
$$

On the other hand, by Schwarz inequality and by 7.2 , on the set $\mathcal{D}_{\boldsymbol{z}}, G(\boldsymbol{x})$ is absolutely bounded by $C_{0} N \delta_{N}$. Putting together the previous two estimates, we get that

$$
\max _{\boldsymbol{x} \in \mathcal{D}_{\boldsymbol{z}}} \mathbf{P}_{\boldsymbol{x}}\left[\mathcal{A}_{N}\right] \leq C_{0}\left(\frac{\delta_{N}}{\varepsilon_{N}^{2}}+\frac{1}{\sqrt{N} \varepsilon_{N}}+\sqrt{N} \varepsilon_{N}^{2}\right) .
$$

This completes the proof of the lemma in view of the definition of the sequence $\varepsilon_{N}$. 
Proof of Proposition 7.2. The proof is similar to the one of the two previous lemmas. Let $g(x)=\int_{0}^{x} \exp \left\{-\mu y^{2} / 2\right\} d y$. By Dynkin's formula, for every $t>0, \boldsymbol{x} \in \mathcal{D}_{\boldsymbol{z}}$, 7.5 holds for $G(\boldsymbol{x})=g\left(N^{1 / 2}[\boldsymbol{x}-\boldsymbol{z}] \cdot \boldsymbol{v}\right)$. Since $g^{\prime \prime}(x)+\mu x g^{\prime}(x)=0$, and since the first three derivative of $g$ are uniformly bounded, by Lemma 7.4 and by (7.4),

$$
\max _{\boldsymbol{x} \in \mathcal{D}_{\boldsymbol{z}}}\left|\mathbf{E}_{\boldsymbol{x}}\left[G\left(X_{N}\left(H_{N}\right)\right)\right]-G(\boldsymbol{x})\right| \leq C_{0} \varepsilon_{N}^{3} N \rightarrow 0 .
$$

On $D_{\boldsymbol{z}}$, the function $G$ vanishes. On the other hand, on the event $\left\{H_{N}=H_{N}^{ \pm}\right\}$, $G\left(X_{N}\left(H_{N}\right)\right)= \pm(2 \pi / \mu)^{1 / 2}+o_{N}(1)$. Therefore, by Lemma 7.5 .

$$
\lim _{N \rightarrow \infty} \max _{\boldsymbol{x} \in \mathcal{D}_{\boldsymbol{z}}}\left|\mathbf{P}_{\boldsymbol{x}}\left[H_{N}=H_{N}^{+}\right]-\mathbf{P}_{\boldsymbol{x}}\left[H_{N}=H_{N}^{-}\right]\right|=0 .
$$

This completes the proof of the proposition in view of Lemma 7.5 .

Proof of Theorem 2.7. Fix $1 \leq i \leq i_{0}, 1 \leq j \leq \ell_{i}$. For each $a \in S$, let $\left\{\boldsymbol{x}_{a}^{N}: N \geq 1\right\}$ be a sequence of points in $\varepsilon_{N}^{a}$. Denote by $\hat{R}_{N}(a, b), a \neq b \in S$, the jump rates of the trace of $X_{N}(t)$ on the set $\left\{\boldsymbol{x}_{N}^{a}: a \in S\right\}$. By [2, Lemma 6.8],

$$
\begin{gathered}
\mu_{N}\left(\boldsymbol{x}_{N}^{a}\right) \hat{R}_{N}(a, b)=\frac{1}{2}\left\{\operatorname{cap}_{N}\left(\left\{\boldsymbol{x}_{N}^{a}\right\}, \hat{S} \backslash\left\{\boldsymbol{x}_{N}^{a}\right\}\right)+\operatorname{cap}_{N}\left(\left\{\boldsymbol{x}_{N}^{b}\right\}, \hat{S} \backslash\left\{\boldsymbol{x}_{N}^{b}\right\}\right)\right. \\
\left.-\operatorname{cap}_{N}\left(\left\{\boldsymbol{x}_{N}^{a}, \boldsymbol{x}_{N}^{b}\right\}, \hat{S} \backslash\left\{\boldsymbol{x}_{N}^{a}, \boldsymbol{x}_{N}^{b}\right\}\right)\right\},
\end{gathered}
$$

where $\hat{S}=\left\{\boldsymbol{x}_{N}^{c}: c \in S\right\}$. By Remark 2.3. equation (2.8), and the fact that $c_{1}\left(a^{\prime}, b^{\prime}\right)=\boldsymbol{c}\left(a^{\prime}, b^{\prime}\right)$, for $a \neq b \in S$,

$$
\lim _{N \rightarrow \infty} \frac{\hat{R}_{N}(a, b)}{\sum_{c \in S, c \neq a} \hat{R}_{N}(a, c)}=p(a, b),
$$

where $p(a, b)$ has been introduced in (2.6).

On the other hand, by [2, Proposition 6.1], for $a \neq b \in S$,

$$
\hat{R}_{N}(a, b)=\lambda_{N}\left(\boldsymbol{x}_{N}^{a}\right) \mathbf{P}_{\boldsymbol{x}_{N}^{a}}\left[H_{\boldsymbol{x}_{N}^{b}}=H_{\hat{S}}^{+}\right],
$$

and by the strong Markov property,

$$
\mathbf{P}_{\boldsymbol{x}_{N}^{a}}\left[H_{\boldsymbol{x}_{N}^{b}}=H_{\hat{S}}^{+}\right]=\mathbf{P}_{\boldsymbol{x}_{N}^{a}}\left[H_{\boldsymbol{x}_{N}^{b}}=H_{\hat{S} \backslash\left\{\boldsymbol{x}_{N}^{a}\right\}}\right] \mathbf{P}_{\boldsymbol{x}_{N}^{a}}\left[H_{\hat{S}}^{+}<H_{\boldsymbol{x}_{N}^{a}}^{+}\right] .
$$

It follows from the last three displayed equations that

$$
\lim _{N \rightarrow \infty} \mathbf{P}_{\boldsymbol{x}_{N}^{a}}\left[H_{\boldsymbol{x}_{N}^{b}}=H_{\hat{S} \backslash\left\{\boldsymbol{x}_{N}^{a}\right\}}\right]=p(a, b), \quad a \neq b \in S .
$$

Since any continuous path from $\boldsymbol{x}_{N}^{a}$ to $\hat{S} \backslash\left\{\boldsymbol{x}_{N}^{a}\right\}$ must cross $D_{a}, H_{\mathcal{D}_{a}}<H_{\hat{S} \backslash\left\{\boldsymbol{x}_{N}^{a}\right\}}$

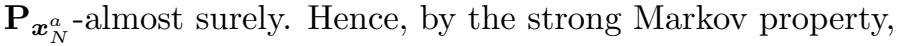

$$
\mathbf{P}_{\boldsymbol{x}_{N}^{a}}\left[H_{\boldsymbol{x}_{N}^{b}}=H_{\hat{S} \backslash\left\{\boldsymbol{x}_{N}^{a}\right\}}\right]=\mathbf{E}_{\boldsymbol{x}_{N}^{a}}\left[\mathbf{P}_{X_{N}\left(H_{\mathcal{D}_{a}}\right)}\left[H_{\boldsymbol{x}_{N}^{b}}=H_{\hat{S} \backslash\left\{\boldsymbol{x}_{N}^{a}\right\}}\right]\right] .
$$

By Lemma 7.1 .

$$
\lim _{N \rightarrow \infty} \mathbf{P}_{\boldsymbol{x}_{N}^{a}}\left[H_{\mathcal{D}_{a}}<H_{\mathcal{B}_{a}}\right]=0
$$

Therefore,

$$
\begin{aligned}
& \lim _{N \rightarrow \infty} \mathbf{P}_{\boldsymbol{x}_{N}^{a}}\left[H_{\boldsymbol{x}_{N}^{b}}=H_{\hat{S} \backslash\left\{\boldsymbol{x}_{N}^{a}\right\}}\right] \\
& \quad=\lim _{N \rightarrow \infty} \sum_{\boldsymbol{z} \in \mathfrak{S}_{a}} \sum_{\boldsymbol{y} \in \mathcal{D}_{\boldsymbol{z}}} \mathbf{P}_{\boldsymbol{x}_{N}^{a}}\left[H_{\mathcal{D}_{a}}=H_{\boldsymbol{y}}\right] \mathbf{P}_{\boldsymbol{y}}\left[H_{\boldsymbol{x}_{N}^{b}}=H_{\hat{S} \backslash\left\{\boldsymbol{x}_{N}^{a}\right\}}\right] .
\end{aligned}
$$


By the strong Markov property at time $H_{\hat{S}}$, the previous expression is equal to

$$
\lim _{N \rightarrow \infty} \sum_{\boldsymbol{z} \in \mathfrak{S}_{a}} \sum_{\boldsymbol{y} \in \mathcal{D}_{\boldsymbol{z}}} \sum_{c \in S} \mathbf{P}_{\boldsymbol{x}_{N}^{a}}\left[H_{\mathcal{D}_{a}}=H_{\boldsymbol{y}}\right] \mathbf{P}_{\boldsymbol{y}}\left[H_{\boldsymbol{x}_{N}^{c}}=H_{\hat{S}}\right] \mathbf{P}_{\boldsymbol{x}_{N}^{c}}\left[H_{\boldsymbol{x}_{N}^{b}}=H_{\hat{S} \backslash\left\{\boldsymbol{x}_{N}^{a}\right\}}\right] .
$$

By Corollary 7.3 this limit is equal to

$$
\begin{aligned}
& \frac{1}{2} \lim _{N \rightarrow \infty} \mathbf{P}_{\boldsymbol{x}_{N}^{a}}\left[H_{\boldsymbol{x}_{N}^{b}}=H_{\hat{S} \backslash\left\{\boldsymbol{x}_{N}^{a}\right\}}\right] \mathbf{P}_{\boldsymbol{x}_{N}^{a}}\left[H_{\mathcal{D}_{a}}=H_{\mathcal{B}_{a}}\right] \\
& \quad+\frac{1}{2} \lim _{N \rightarrow \infty} \sum_{\boldsymbol{z} \in \mathfrak{S}_{a, b}} \mathbf{P}_{\boldsymbol{x}_{N}^{a}}\left[H_{\mathcal{D}_{a}}=H_{\mathcal{D}_{\boldsymbol{z}}}\right] .
\end{aligned}
$$

By (7.7), we may replace in the first line $\mathbf{P}_{\boldsymbol{x}_{N}^{a}}\left[H_{\mathcal{D}_{a}}=H_{\mathcal{B}_{a}}\right]$ by 1 .

In conclusion, in view of (7.6), we have shown that

$$
p(a, b)=\lim _{N \rightarrow \infty} \mathbf{P}_{\boldsymbol{x}_{N}^{a}}\left[H_{\boldsymbol{x}_{N}^{b}}=H_{\hat{S} \backslash\left\{\boldsymbol{x}_{N}^{a}\right\}}\right]=\lim _{N \rightarrow \infty} \sum_{\boldsymbol{z} \in \mathfrak{S}_{a, b}} \mathbf{P}_{\boldsymbol{x}_{N}^{a}}\left[H_{\mathcal{D}_{a}}=H_{\mathcal{D}_{\boldsymbol{z}}}\right] .
$$

This completes the proof of the theorem in the case where the set $\mathfrak{S}_{a, b}$ is a singleton. It is not difficult to modify this argument to handle the case with more than one saddle point between two wells. Indeed, since the proof does not depend on the behavior of the function $F$ on $W_{a}^{c}$, we can modify $F$ on $W_{a}^{c} \backslash\left[\cup_{\boldsymbol{z} \in \mathfrak{S}_{a}} B_{\epsilon}(\boldsymbol{z})\right]$, for some $\epsilon>0$, creating new wells of height $H_{i}$, and turning each saddle point $\boldsymbol{z} \in \mathfrak{S}_{a}$ the unique saddle point between the well $W_{a}$ and new well $W_{\boldsymbol{z}}^{\prime}$.

\section{REFERENCES}

[1] L. Avena, A. Gaudillière: On some random forests with determinantal roots. arXiv: $1310.1723 \mathrm{v} 3$

[2] J. Beltrán, C. Landim: Tunneling and metastability of continuous time Markov chains. J. Stat. Phys. 140, 1065-1114, (2010).

[3] J. Beltrán, C. Landim; Tunneling and metastability of continuous time Markov chains II. J. Stat. Phys. 149, 598-618 (2012).

[4] J. Beltrán, C. Landim: A Martingale approach to metastability. To appear in Probab. Theory Relat. Fields (2014).

[5] A. Bianchi, A. Bovier, D. Ioffe: Sharp asymptotics for metastability in the random field Curie-Weiss model. Electron. J. Probab. 14, 1541-1603, (2009).

[6] A. Bianchi, A. Bovier, D. Ioffe: Pointwise estimates and exponential laws in metastable systems via coupling methods. Ann. Probab. 40, 339-371, (2012).

[7] A. Bovier, M. Eckhoff, V. Gayrard, M. Klein: Metastability in stochastic dynamics of disordered mean field models. Probab. Theory Relat. Fields 119, 99-161 (2001).

[8] A. Bovier, M. Eckhoff, V. Gayrard, M. Klein: Metastability and low-lying spectra in reversible Markov chains. Comm. Math. Phys. 228, 219-255 (2002).

[9] A. Bovier, M. Eckhoff, V. Gayrard, M. Klein: Metastability in reversible diffusion processes. I. Sharp asymptotics for capacities and exit times. J. Eur. Math. Soc. 6, 399-424 (2004).

[10] A. Bovier, V. Gayrard, M. Klein: Metastability in reversible diffusion processes. II. Precise asymptotics for small eigenvalues. J. Eur. Math. Soc. 7, 69-99 (2005).

[11] M. Cameron, E. Vanden-Eijnden: Flows in Complex Networks: Theory, Algorithms, and Application to LennardJones Cluster Rearrangement. J. Stat. Phys. 156, 427-454 (2014).

[12] M. Cassandro, A. Galves, E. Olivieri, M. E. Vares. Metastable behavior of stochastic dynamics: A pathwise approach. J. Stat. Phys. 35, 603-634 (1984).

[13] W. E, E. Vanden-Eijnden: Towards a theory of transition paths. J. Stat. Phys. 123, 503-523 (2006).

[14] M. I. Freidlin, A. D. Wentzell: Random perturbations of dynamical systems. Translated from the 1979 Russian original by Joseph Szücs. Second edition. Grundlehren der Mathematischen Wissenschaften [Fundamental Principles of Mathematical Sciences], 260. SpringerVerlag, New York, 1998. 
[15] A. Galves, E. Olivieri, M. E. Vares: Metastability for a Class of Dynamical Systems Subject to Small Random Perturbations. Ann. Probab. 15, 1288-1305 (1987).

[16] A. Gaudillière. Condenser physics applied to Markov chains: A brief introduction to potential theory. Online available at http://arxiv.org/abs/0901.3053

[17] H. A. Kramers: Brownian motion in a field of force and the diffusion model of chemical reactions. Physica 7, 284-304 (1940)

[18] C. Landim: A topology for limits of Markov chains. To appear in Stochastic Process. Appl. arXiv:1310.3646 (2013).

[19] P. Metzner, Ch. Schütte, E. Vanden-Eijnden: Transition path theory for Markov jump processes. SIAM Multiscale Model. Simul. 7, 1192-1219 (2009).

[20] F. Noé H. Wu, J. H. Prinz, N. Plattner: Projected and Hidden Markov Models for calculating kinetics and metastable states of complex molecules. arxiv 1309.3220v1

impa, Estrada Dona Castorina 110, CEP 22460 Rio de Janeiro, Brasil and CNRS UMR 6085, Université de Rouen, Avenue de l'Université, BP.12, Technopôle du MadrilLET, F76801 SAint-Étienne-Du-Rouvray, France.

E-MAIL: landim@impa.br

impa, Estrada Dona Castorina 110, CEP 22460 Rio de Janeiro, Brasil.

E-MAIL: misturini@impa.br

Graduate School of Mathematical Sciences, The University of Tokyo, Komaba, TOKYO 153-8914, JAPAN.

E-MAIL: tsunoda@ms.u-tokyo.ac.jp 